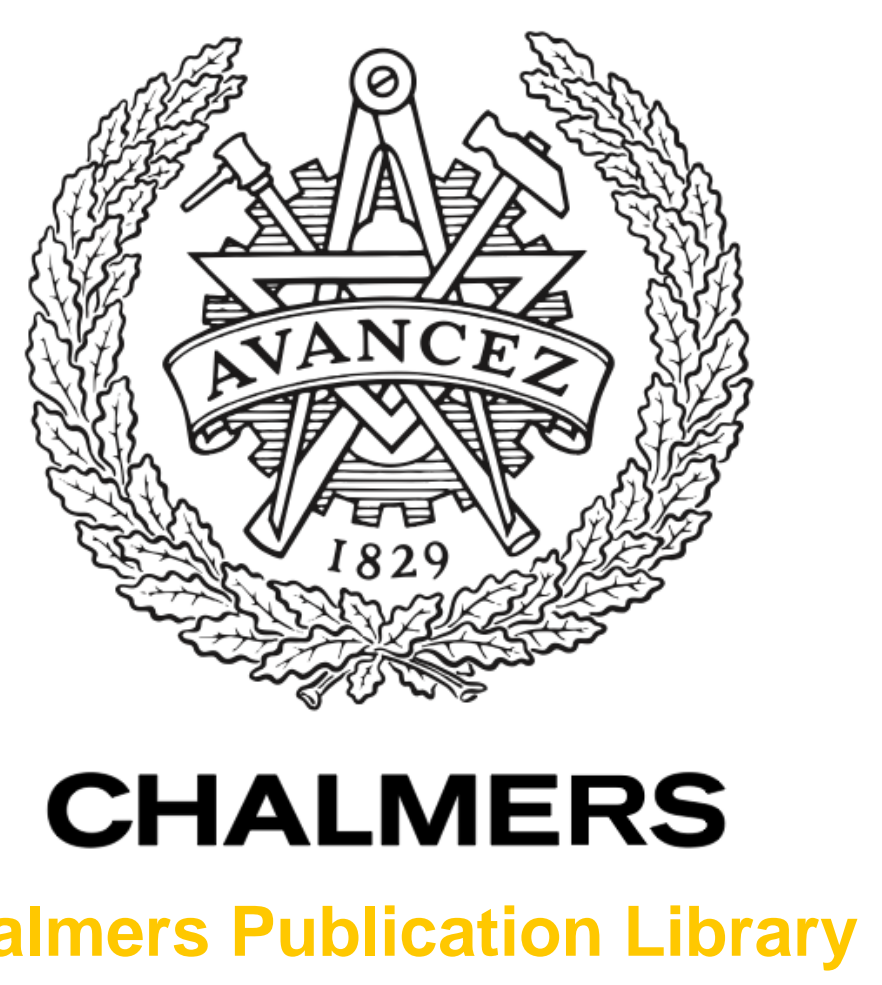

Chalmers Publication Library

\title{
An Alma Survey of Sub-Millimeter Galaxies in the Extended Chandra Deep Field South: Sub-Millimeter Properties of Color-Selected Galaxies
}

This document has been downloaded from Chalmers Publication Library (CPL). It is the author's version of a work that was accepted for publication in:

\section{Astrophysical Journal (ISSN: 0004-637X)}

Citation for the published paper:

Decarli, R. ; Smail, I. ; Walter, F. (2014) "An Alma Survey of Sub-Millimeter Galaxies in the Extended Chandra Deep Field South: Sub-Millimeter Properties of Color-Selected Galaxies". Astrophysical Journal, vol. 780(2),

http://dx.doi.org/10.1088/0004-637X/780/2/115

Downloaded from: http://publications.lib.chalmers.se/publication/193789

Notice: Changes introduced as a result of publishing processes such as copy-editing and formatting may not be reflected in this document. For a definitive version of this work, please refer to the published source. Please note that access to the published version might require a subscription. 


\title{
AN ALMA SURVEY OF SUB-MILLIMETER GALAXIES IN THE EXTENDED CHANDRA DEEP FIELD SOUTH: SUB-MILLIMETER PROPERTIES OF COLOR-SELECTED GALAXIES
}

\author{
R. Decarli ${ }^{1}$, I. Smail ${ }^{2}$, F. Walter ${ }^{1}$, A. M. Swinbank ${ }^{2}$, S. Chapman ${ }^{3,4}$, K. E. K. Coppin ${ }^{5}$, P. Cox $^{6,7}$,

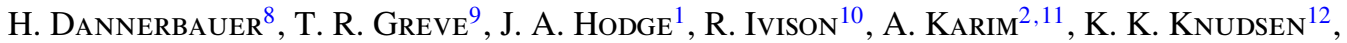 \\ L. Lindroos ${ }^{12}$, H.-W. Rix ${ }^{1}$, E. SChinNERER ${ }^{1}$, J. M. Simpson ${ }^{2}$, P. van DER WerF ${ }^{13}$, AND A. Wei ${ }^{14}$ \\ ${ }^{1}$ Max-Planck Institut für Astronomie, Königstuhl 17, D-69117 Heidelberg, Germany; decarli@ mpia.de \\ 2 Institute for Computational Cosmology, Durham University, South Road, Durham DH1 3LE, UK \\ ${ }^{3}$ Institute of Astronomy, University of Cambridge, Madingley Road, Cambridge CB3 OHA, UK \\ ${ }^{4}$ Department of Physics and Atmospheric Science, Dalhousie University, Coburg Road Halifax B3H 4R2, UK \\ ${ }^{5}$ Centre for Astrophysics, Science and Technology Research Institute, University of Hertfordshire, Hatfield AL10 9AB, UK \\ ${ }^{6}$ IRAM, 300 rue de la piscine, F-38406 Saint-Martin d'Hères, France \\ 7 ALMA, Alonso de Córdova 3107, Vitacura, Santiago, Chile \\ ${ }^{8}$ Universität Wien, Institut für Astrophysik, Türenschanzstrasse 17, A-1180 Wien, Austria \\ ${ }^{9}$ University College London, Department of Physics and Astronomy, Gower Street, London WC1E 6BT, UK \\ ${ }^{10}$ Institute for Astronomy, University of Edinburgh, Blackford Hill, Edinburgh EH9 3HJ, UK \\ ${ }^{11}$ Argelander-Institute of Astronomy, Bonn University, Auf dem Hügel 71, D-53121 Bonn, Germany \\ 12 Department of Earth and Space Sciences, Chalmers University of Technology, Onsala Space Observatory, Onsala SE-439 92, Sweden \\ ${ }^{13}$ Leiden Observatory, Leiden University, P.O. Box 9513, 2300 RA Leiden, Netherlands \\ ${ }^{14}$ Max-Planck Institut für Radioastronomie, Auf dem Hügel 69, D-53121 Bonn, Germany \\ Received 2013 August 21; accepted 2013 November 11; published 2013 December 16
}

\begin{abstract}
We study the sub-millimeter properties of color-selected galaxies via a stacking analysis applied for the first time to interferometric data at sub-millimeter wavelengths. We base our study on $344 \mathrm{GHz}$ ALMA continuum observations of $\sim 20^{\prime \prime}$-wide fields centered on 86 sub-millimeter sources detected in the LABOCA Extended Chandra Deep Field South (ECDFS) Sub-millimeter Survey. We select various classes of galaxies ( $K$-selected, star-forming sBzK galaxies, extremely red objects, and distant red galaxies) according to their optical/near-infrared fluxes. We find clear, $>10 \sigma$ detections in the stacked images of all these galaxy classes. We include in our stacking analysis Herschel/SPIRE data to constrain the dust spectral energy distribution of these galaxies. We find that their dust emission is well described by a modified blackbody with $T_{\text {dust }} \approx 30 \mathrm{~K}$ and $\beta=1.6$ and infrared luminosities of $(5-11) \times 10^{11} L_{\odot}$ or implied star formation rates of 75-140 $M_{\odot} \mathrm{yr}^{-1}$. We compare our results with those of previous studies based on single-dish observations at $870 \mu \mathrm{m}$ and find that our flux densities are a factor 2-3 higher than previous estimates. The discrepancy is observed also after removing sources individually detected in ALESS maps. We report a similar discrepancy by repeating our analysis on $1.4 \mathrm{GHz}$ observations of the whole ECDFS. Hence, we find tentative evidence that galaxies that are associated in projected and redshift space with sub-mm bright sources are brighter than the average population. Finally, we put our findings in the context of the cosmic star formation rate density as a function of redshift.
\end{abstract}

Key words: galaxies: high-redshift - galaxies: star formation - submillimeter: galaxies techniques: interferometric

Online-only material: color figures

\section{INTRODUCTION}

A variety of tracers are used to probe star formation in distant galaxies based on rest-frame ultraviolet luminosities, optical colors, recombination line luminosities, polycyclic aromatic hydrocarbon features, dust luminosity, and radio emission (e.g., Condon 1992; Kennicutt 1998; Kewley et al. 2001; Yun \& Carilli 2002; Bell 2003; Brinchmann et al. 2004; Daddi et al. 2007; Salim et al. 2007; Sargent et al. 2010; Karim et al. 2011; Leroy et al. 2012; Murphy et al. 2012). The dust continuum luminosity is of particular interest in the study of star formation in high- $z$ galaxies: as the dust spectral energy distribution (SED) shifts to higher and higher redshifts, we observe closer and closer to the peak of the dust emissivity at sub-millimeter wavelengths. This negative $k$-correction is such that it roughly compensates for the flux dimming due to the increased luminosity distance, so that a galaxy with a fixed infrared (IR) luminosity will show about the same sub-millimeter flux density at any redshift $1<z<6$ (e.g., Blain et al. 2002).
Sub-millimeter observations are thus especially suited to study star formation in high- $z$ galaxies. However, with the exception of a few strongly lensed cases (e.g., Knudsen et al. 2009; Swinbank et al. 2010), so far only the very bright end of the IR luminosity function has been constrained (e.g., Barger et al. 1999; Borys et al. 2003; Coppin et al. 2006; Weiß et al. 2009; Austermann et al. 2010) because of sensitivity and resolution limits of single-dish observations. These bright (several mJy at $350 \mathrm{GHz}$ ) sources show redshift distributions peaking around $z \gtrsim 2$, IR luminosities exceeding $10^{12} L_{\odot}$, and associated star-formation rates (SFRs) of hundreds or thousands of solar masses per year (Chapman et al. 2003, 2005; Solomon \& Vanden Bout 2005; Walter 2009; Hatsukade et al. 2010; Wardlow et al. 2011; Moncelsi et al. 2011; Walter et al. 2012; Simpson et al. 2014; Swinbank et al. 2014). However, these sources are not representative of the more common star-forming galaxies, with SFRs of $\lesssim 10 M_{\odot} \mathrm{yr}^{-1}$ (see, e.g., Da Cunha et al. 2013), which dominate the cosmic SFR density (SFRD). In order to sample these sources, sensitivities of $\lesssim 0.1 \mathrm{mJy}$ at $350 \mathrm{GHz}$ are required. 
These depths are expensive to achieve (in terms of observing time) even with the full ALMA (e.g., in order to obtain a $1 \sigma$ sensitivity of $10 \mu \mathrm{Jy}$ at $344 \mathrm{GHz}$ in a continuum observation with 50 antennas, one needs $\sim 3.6 \mathrm{hr}$ on-source). Moreover, ALMA observations at (sub-)millimeter wavelengths cover only a small region on the sky (the primary beam diameter is $17^{\prime \prime} .3$ at $344 \mathrm{GHz}$ ). Therefore, many pointings are required in order to address cosmic variance.

A common way to push the sensitivity of observations of a class of faint sources is through stacking analysis of galaxies selected, e.g., via their optical/near-IR (NIR) emission. Various studies have applied stacking techniques basically at any wavelength: $\gamma$-rays (e.g., Aleksić et al. 2011), X-rays (Chaudhary et al. 2012; George et al. 2012), optical/NIR (Zibetti et al. 2007; Matsuda et al. 2012; González et al. 2012), mid-IR/far-IR (Dole et al. 2006; Kurczynzki \& Gawiser 2010; Bourne et al. 2012), sub-millimeter (Webb et al. 2004; Knudsen et al. 2005; Greve et al. 2010), and radio (e.g., Boyle et al. 2007; Ivison et al. 2007; Hodge et al. 2008, 2009; Dunne et al. 2009; Fabello et al. 2011; Karim et al. 2011). In particular, Greve et al. (2010, hereafter, G10) undertook a stacking analysis of the LABOCA Extended Chandra Deep Field South Sub-millimeter Survey (LESS; Weiß et al. 2009), a $870 \mu \mathrm{m}$ (344 GHz) survey of a $30^{\prime} \times 30^{\prime}$ wide region around the Extended Chandra Deep Field South (ECDFS; Giacconi et al. 2001), also encompassing GOODS-South and the Hubble Ultra Deep Field. They stacked $344 \mathrm{GHz}$ measurements obtained with LABOCA at the positions corresponding to galaxies grouped on the basis of their optical/ NIR fluxes and colors and their redshifts. Thanks to the large areal coverage of LESS, several hundred galaxies could be stacked in each galaxy class, thus boosting the sensitivity by more than an order of magnitude, down to few tens of $\mu \mathrm{Jy}$. The major limit of the stacking analysis in G10 is that LABOCA observations have an intrinsic resolution of 19'2. This implies that source blending is a major issue (see Swinbank et al. 2014). A sophisticated deblending algorithm based on neighbor chains was applied in G10 (see also Kurczynzki \& Gawiser 2010).

Here, we build up on the analysis by G10 and perform a similar stacking analysis on new interferometric ALMA observations of the fields encompassing LESS-detected galaxies (the ALESS survey: Hodge et al. 2013; Karim et al. 2013, see Section 2). These data have been collected at the same (effective) frequency of the original LESS observations and reach typical a rms of $\approx 0.4 \mathrm{mJy}$ beam $^{-1}$, i.e., a factor of three better than LESS. Most importantly, these interferometry observations have typical beam sizes of $\sim 1^{\prime \prime} .6 \times 1$ 1. 15 , i.e., $\sim 200$ times smaller than in the LABOCA single-dish observations (in terms of beam area). This matches the typical angular size of galaxies at high- $z$, so source blending is no longer an issue at this frequency. This enormously simplifies the interpretation of the results of any stacking analysis.

The structure of this paper is as follows. Section 2 briefly introduces the ALMA survey of LESS-detected sources (ALESS) data. Section 3 describes the galaxy catalog and the color cuts used to define the stacked samples. We explain our stacking routine in Section 4. In Section 5, we present the results of our analysis, we infer IR luminosities and SFR estimates, and we compare our findings with previous works. Our conclusions are drawn in Section 6. Throughout the paper, we will assume a standard cosmology model with $H_{0}=70 \mathrm{~km} \mathrm{~s}^{-1} \mathrm{Mpc}^{-1}$, $\Omega_{\mathrm{m}}=0.3$, and $\Omega_{\Lambda}=0.7$. All magnitudes refer to the AB photometric system (Oke 1974), unless specified.

\section{THE ALESS DATA}

Our analysis is based on the ALESS. These are $344 \mathrm{GHz}$ observations of 122 sources. Observations were carried out during ALMA Cycle 0 ("Early Science") between 2011 October 18 and November 3 with the array in ALMA Cycle 0's compact configuration (longest baseline: $125 \mathrm{~m}$ ), mostly with 15 antennas. The typical resolution element has a FWHM $\sim 1^{\prime \prime}$. $6 \times 1^{\prime \prime}$. 15 . At $344 \mathrm{GHz}$, the full width at half power of the ALMA primary beam is $17^{\prime \prime} .3$ (scaling as $19^{\prime \prime} .9 \times(300 / v)$, where $v$ is the observing frequency in $\mathrm{GHz}$ ). The data were reduced and cleaned down to a $3 \sigma$ level using the Common Astronomy Software Application. Details on the survey design, the data reduction, and the cleaning process are described in Hodge et al. (2013). Final maps are $128 \times 128$ pixel, with a pixel scale of $0^{\prime \prime} .2$ pixel $^{-1}$. The typical central rms of the final maps is $\approx 0.4 \mathrm{mJy}^{\text {beam }}{ }^{-1}$. In our analysis, we focus only on the 86 "good quality" maps with rms $<0.6 \mathrm{mJy}^{\text {beam }}{ }^{-1}$ and beam axis ratios $<2$ (see Hodge et al. 2013 for details).

\section{THE OPTICAL/NIR DATASET}

We base our analysis on the photometric catalog by Simpson et al. (2014), who capitalized on deep, optical/NIR archival data from various surveys of the ECDFS. The bulk of the data are taken from the Wide MUlti-wavelength Survey by Yale-Chile (MUSYC; Taylor et al. 2009, hereafter T09). For more details on MUSYC, see Gawiser et al. (2006). The MUSYC catalog consists of $16,910 \mathrm{~K}$-band flux-limited sources in the $30^{\prime} \times 30^{\prime}$ wide region at the center of the ECDFS. It provides optical and NIR photometry in the UBVRIzJHK bands. At $K_{\mathrm{AB}}=22 \mathrm{mag}$, the catalog is $100 \%$ complete for point sources and $96 \%$ complete for sources with a scale radius of $\approx 0.5(\approx 4.2 \mathrm{kpc}$ at $z=2)$. This corresponds to a stellar mass $\left(M_{*}\right)$ completeness of $>90 \%$ at $z=1.8$ for $M_{*}>10^{11} M_{\odot}$ (T09). Following G10, we focus on the $K_{\text {Vega }}<20 \mathrm{mag}$ (i.e., $K_{\mathrm{AB}}<21.83 \mathrm{mag}$ ) galaxies to ease the comparison with previous studies. We take into account flux aperture corrections by scaling all the fluxes according to the SExtractor-to-total flux ratio in the $K$ band, as provided in T09. Additional photometric data in Simpson et al. (2014) include deep $J$ - and $K$-band images from $\mathrm{S}$. Zibetti et al. (in preparation), the Taiwan ECDFS NIR survey (Hsieh et al. 2012), and Spitzer/IRAC 3.6, 4.5, 5.8, and $8.0 \mu \mathrm{m}$ images from the Spitzer IRAC/MUSYC Public Legacy Survey (Damen et al. 2011).

Simpson et al. (2014) performed SED fitting and photometric redshift estimates for all the sources in an IRAC $3.6 \mu \mathrm{m}$-selected catalog of the ECDFS. Once compared with the spectroscopic redshifts, the typical accuracy is $\Delta z /(1+z)=0.011$. In the following analysis, we will refer to the best redshift estimate (spectroscopic if available) for all the sources.

The photometric catalog is used to select galaxies from stars and split galaxies in various subsamples, as follows.

1. Galaxies with $K_{\text {Vega }}<20 \mathrm{mag}$, separated from stars by requiring $(z-K-0.04)>0.3 \times(B-z+0.56)-0.5$ and $(J-K)>0.1$, following Daddi et al. (2004) and G10.

2. Actively star-forming galaxies at $1.4<z<2.5$. These objects are called sBzK galaxies and they are defined by requiring $(z-K-0.04)-(B-z+0.56)>-0.2$ (Daddi et al. 2004).

3. Extremely red objects (EROs), defined as galaxies with $(R-K)>3.35$ and $(J-K)>0.1$ (Elston et al. 1988).

4. Distant red galaxies (DRGs), defined as $(J-K)>1.32$ (Franx et al. 2003). 

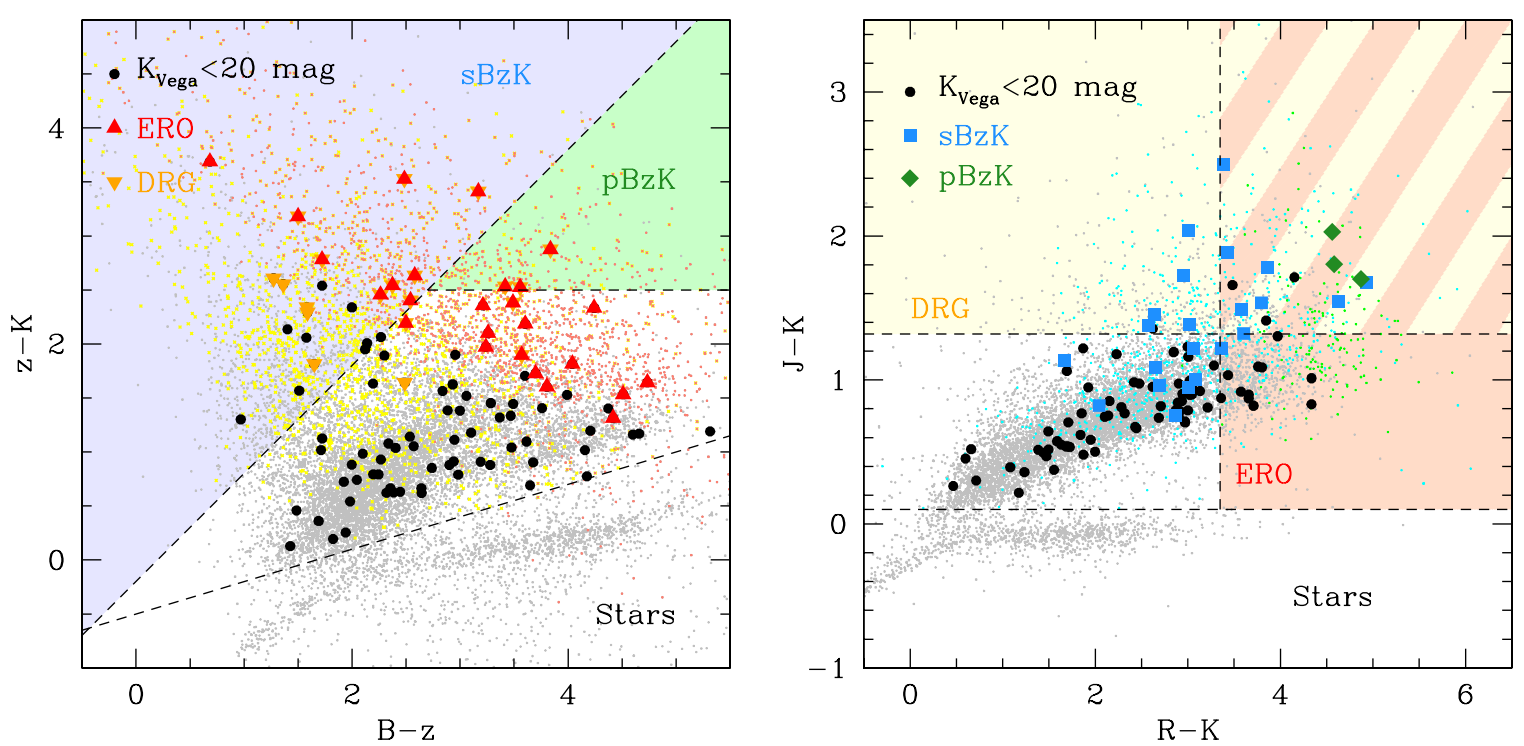

Figure 1. Optical/NIR color-color diagrams used to define the different stacking samples of galaxies. Dots are sources in the photometric catalog. Circles, triangles, squares, and diamonds highlight sources that lie within the primary beam of ALMA pointings $\left(\sim 20^{\prime \prime}\right.$ in diameter $)$ used in this study. Left: the BzK diagnostic allows us to identify stars from galaxies and to isolate $1.4 \lesssim z \lesssim 2.5$ star-forming and passive galaxies (top-left and top-right corners, respectively). EROs and DRGs are highlighted with red and yellow points (upward/downward triangles), respectively. Right: the $(R-K, J-K)$ color diagram allows us to distinguish stars from galaxies and is used to define EROs and DRGs. Blue and green symbols (squares/diamonds) show how sBzK and pBzK galaxies distribute over this plot.

(A color version of this figure is available in the online journal.)

All the galaxies selected as sBzK, EROs, or DRGs are also part of the $K_{\text {Vega }}<20$ mag sample.

Figure 1 shows key color cuts adopted in the definitions of our galaxy samples. The line defining sBzK galaxies runs parallel with the reddening vector, so that galaxies move up and to the right with increasing reddening in the left-hand panel of Figure 1. This selects massive, star-forming galaxies at $1.4<z<2.5$ irrespective of dust extinction (as long as they pass the $K$-band flux selection). In the same plot, a $z>1.4$ galaxy with fixed reddening would move up and to the right as its stellar population grows older. Therefore, the wedge defined by requiring $(z-K-0.04)-(B-z+0.56)<-0.2$ and $(z-K-0.04)>2.5$ is populated by passive, old galaxies, dubbed passive BzK galaxies (pBzK; Daddi et al. 2004). On the other hand, the NIR color cuts used to identify EROs and DRGs select objects with very red colors, either intrinsic (i.e., associated with passive, old stellar populations at high $z$ ) or due to reddening (Daddi et al. 2004; Lane et al. 2007; Greve et al. 2010), therefore these galaxies include high- $z$ sources with old stellar population (including pBzK galaxies) or highly obscured star-forming galaxies.

There is clear overlap in the sample definitions. In particular, pBzK galaxies are a subsample of the ERO class; about one-third of the sBzK-selected galaxies are also DRGs or EROs, where the fraction depends on redshift and NIR flux (most of the sBzK galaxies classified also as EROs or DRGs reside at $z>2$ and are faint in the $K$ band); about three-fourths of the DRGs are also selected as EROs. In the remainder of our analysis, we will not include $\mathrm{pBzK}$ galaxies, as the total number of $\mathrm{pBzK}$ covered in the ALESS observations is only three.

Figure 2 shows the redshift and NIR luminosity distributions of the galaxies in each sample. NIR luminosities are computed by interpolating the best SED fits from Simpson et al. (2014). $K$-band-selected galaxies span a wide range in redshift, from $0-2.6$, while color selection efficiently identify sources with $1<z<2.6$. Lane et al. (2007) suggested that DRGs can have a broader redshift distribution than EROs, but this is not observed in our sample. Similarly, $K$-selected galaxies show a broad range of rest-frame $H$-band luminosities (from -20 to $-26 \mathrm{mag}$ ), while color-selected galaxies tend to be bright $\left(M_{\mathrm{H}}<-23 \mathrm{mag}\right)$.

\section{THE STACKING ROUTINE}

\subsection{Method}

Our stacking routine works as follows: (1) from the photometric catalog, we select galaxies according to their fluxes and colors (as described in Section 3). We keep only sources lying within $1.2 \times$ the primary beam radius of each pointing (i.e., within 10 ' 4 from each pointing center), i.e., where the sensitivity is $>1 / 3$ of that at the pointing center. Out of 86 "good quality" ALESS pointings, 74 overlap with the area covered by the MUSYC catalog and 55 pointings contain at least 1 galaxy belonging to one of the classes defined in Section 3. (2) We compute the offset of each galaxy with respect to the pointing center of the ALMA observations. This distance is used to compute the primary beam correction, modeled as $\exp \left(\operatorname{offset}^{2} /\left(2 \sigma_{\mathrm{PB}}^{2}\right)\right)$, where $\sigma_{\mathrm{PB}}=\mathrm{PB} /(2 \sqrt{2 \ln 2})$ and $\mathrm{PB}$ is the primary beam size. (3) For each source, we create a postage stamp from the ALMA $344 \mathrm{GHz}$ image. (4) We align all the postage stamps, scale them to account for the primary beam attenuation computed at step 2 , and then weight average them. Weights are computed as the squared inverse of the primary beam correction computed at the center of each postage stamp ${ }^{15}$ so that a source lying at the primary beam radius has a primary beam correction of two and a weight of 0.25 .

We perform our stacking analysis first on all the galaxies in each bin; then, in order to attempt to account for biases in the sample selection, we exclude those sources that have $344 \mathrm{GHz}$ fluxes brighter than $S_{v}(344 \mathrm{GHz})=3.6 \mathrm{mJy}$ in our ALMA

\footnotetext{
15 Our working assumption is that all the maps have the same depth, which is true to within $\sim 20 \%$ accuracy, given that we consider only the "good quality" maps (see Figure 1 in Hodge et al. 2013).
} 

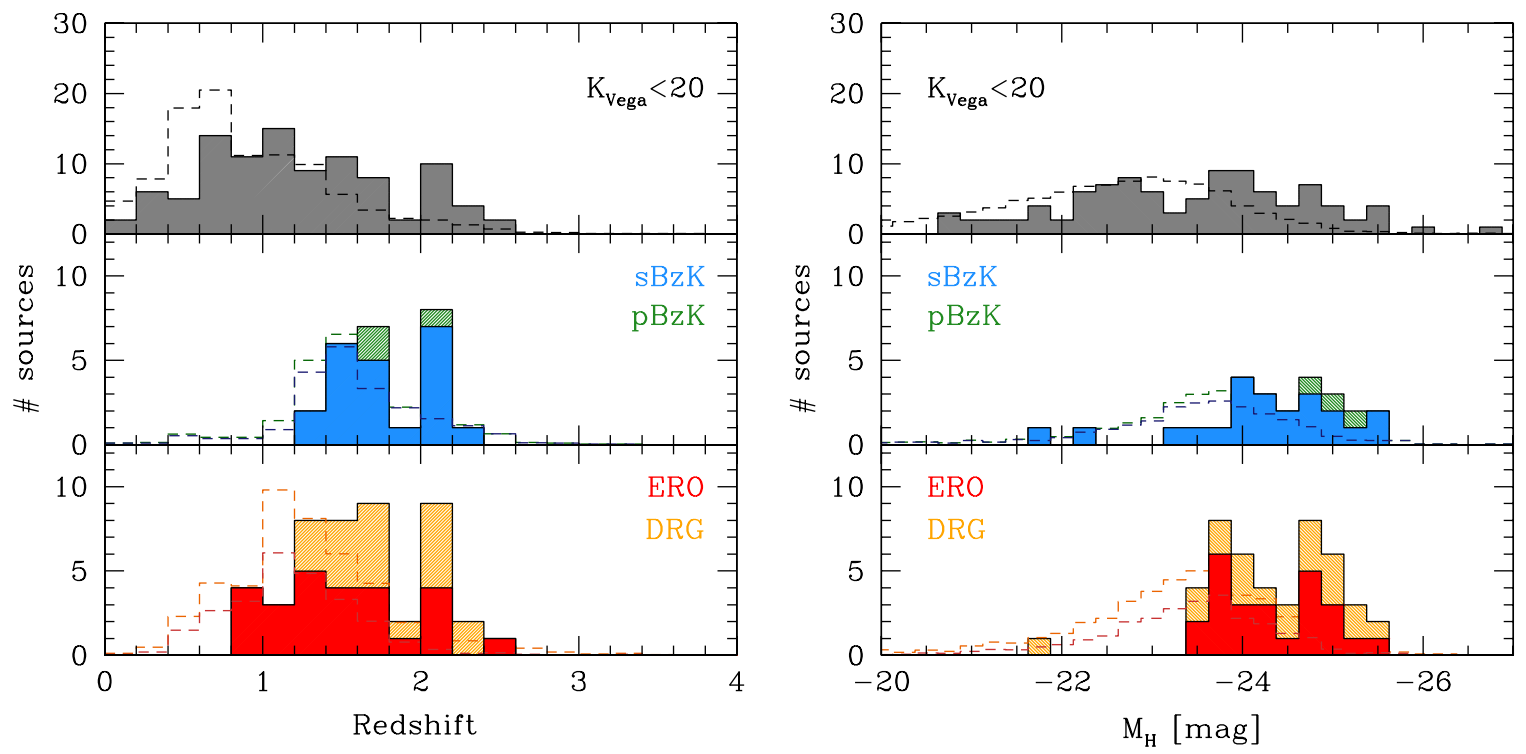

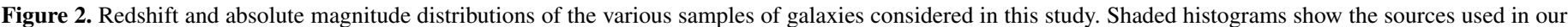

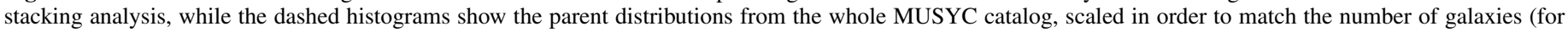

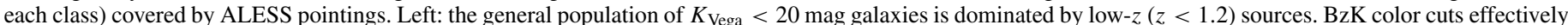

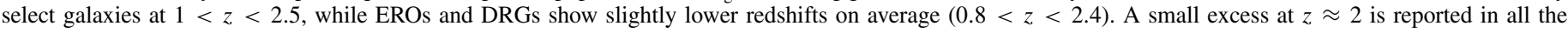

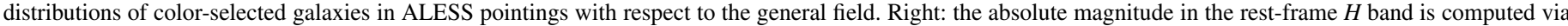

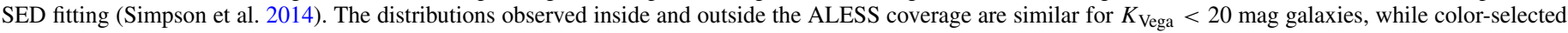
galaxies in our analysis tend to be brighter in the rest-frame $H$ band than their parent samples from the general photometric catalog.

(A color version of this figure is available in the online journal.)

observations, i.e., sources that were detected at $>3 \sigma$ in the original LESS observations. We re-perform the stacks on this "sub-millimeter faint" sample. We then progressively lower the flux cutoff to $S_{v}(344 \mathrm{GHz})=1.8 \mathrm{mJy}$ and $S_{v}(344 \mathrm{GHz})=$ $1.2 \mathrm{mJy}$ and repeat the experiment. The first of these lower flux thresholds tentatively mimics the case where a LESS $3 \sigma$ detection resolved into two sources in our interferometric observations, ${ }^{16}$ consistent with the finding that the bright end of the sub-millimeter galaxy luminosity function observed in the original LESS data is dominated by pairs or multiplets of galaxies that are unresolved in single-dish observations (Karim et al. 2013; Hodge et al. 2013). The second flux cut corresponds to $\mathrm{a} \approx 3 \sigma$ detection in all the ALMA maps considered here, i.e., this subsample excludes any individually detected ALESS source as well. Finally, we consider again the whole dataset (with no $S_{v}(344 \mathrm{GHz})$ cutoff) and perform (1) stacking of only those sources lying at $z>1$ and (2) median stacking instead of weighted averages of all the sources. These different tests allow us to quantify the role of outliers in our final stacks. The analysis is repeated with two different stacking routines, developed independently within our collaboration, and we find consistent results. We have also tested the effects of beam variations among different ALESS pointings by artificially lowering the spatial resolution of all the maps to a circular resolution element with FWHM $=1$ 1'6. The results of this test are in agreement with those obtained when stacking maps in their original resolution (see Table 1).

The distributions of $344 \mathrm{GHz}$ fluxes of the galaxies considered in this study, as measured in the ALESS data, and the results from the stacks are shown in Figure 3. All the samples show a bell-shaped flux distribution with a tail toward positive fluxes,

\footnotetext{
${ }^{16}$ In this simplified approach, we assume equal flux splitting between the two
} sources. implying significant detections from the stacks of each sample (even after excluding the brightest sources).

\subsection{Estimate of Uncertainties}

In order to quantify the uncertainties and biases in the results of our stacking analysis, we repeat our analysis at random positions uniformly distributed over the same area. The assumption here is that the maps are mostly "source-free," so that stacking random positions in the sky corresponds to a random sampling of the noise properties in the maps. For each galaxy set of $N$ sources, we create $N$ random coordinates. The distribution of sources over the various pointings is the same as for the original galaxies (i.e., if a pointing contains three sBzK galaxies, we take three random coordinates from that pointing). We stack the ALESS images of these $N$ random coordinates following the same procedure as in the case of "real" sources. Then, we repeat the whole procedure with a new realization of $N$ random coordinates. We perform 50 iterations in order to gauge the variance of the random stacks. The rms of the distribution of the final stacked fluxes is taken as a measure of the noise in the maps. ${ }^{17}$ We obtain the following rms values: $0.06 \mathrm{mJy}$ beam $^{-1}$,

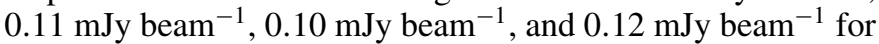
$K_{\text {Vega }}<20$ mag, sBzK, EROs, and DRGs, respectively, when weighted averages are used. Consistent values (within $\approx 10 \%$ ) are found with median stacks. These values are also found to be in good agreement with the pixel rms computed close to the center of stacked images of the real sources: $0.06 \mathrm{mJy}_{\text {beam }}{ }^{-1}$,

\footnotetext{
17 If real sources were always close to the pointing center, the approach outlined here would underestimate the signal-to-noise of the stacking results, due to the primary beam correction. This effect can be estimated a posteriori (by measuring the average attenuation due to primary beam tapering in the samples of real sources and in the case of uniform distributions). The signal-to-noise ratio is underestimated by $3 \%-7 \%$ in the various subsamples, i.e., the correction is negligible for the degree of accuracy required in our analysis.
} 


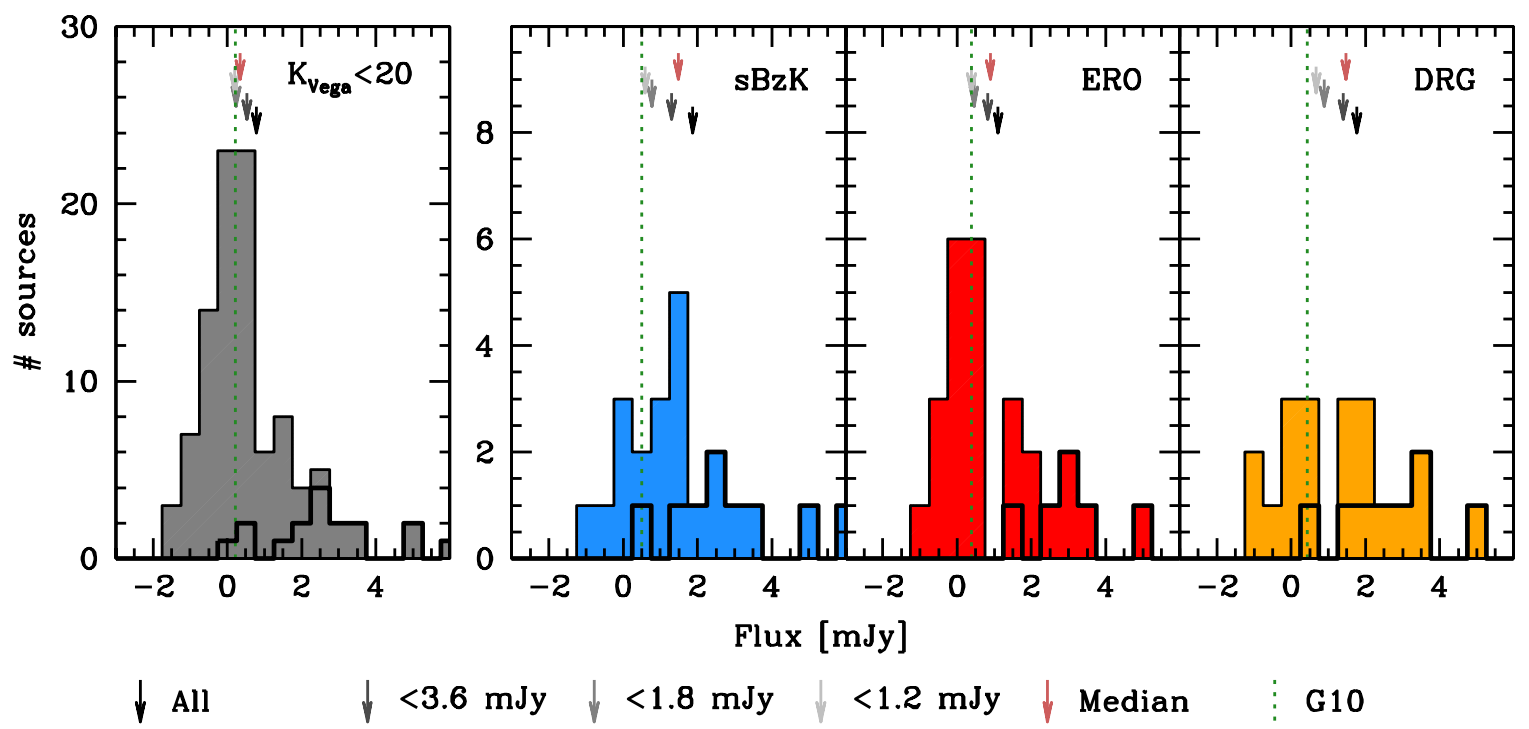

Figure 3. Flux distribution (at $344 \mathrm{GHz}$ ) of individual galaxies considered in this study (shaded histograms). We plot here the flux density value as measured at the positions given in the optical/NIR catalog, after correcting for primary beam attenuation. Thick lines highlight the flux distributions of galaxies physically associated with SMGs (i.e., with consistent photometric redshifts and $<200 \mathrm{kpc}$ separations on the sky). The flux measured from the stacking is shown as arrows, in different colors for different subsamples (see the labels and the text for details). For a comparison, we plot the fluxes obtained in the stacking analysis of LABOCA sources in the full ECDFS presented in G10 as dashed lines.

(A color version of this figure is available in the online journal.)

$0.15 \mathrm{mJy}_{\text {beam }}{ }^{-1}, 0.09 \mathrm{mJy}_{\text {beam }}{ }^{-1}$, and $0.13 \mathrm{mJy}_{\text {beam }}{ }^{-1}$ for $K_{\text {Vega }}<20$ mag, sBzK, EROs, and DRGs, respectively. Finally, we have verified that the average stacked values in the random iterations are always consistent with zero.

\subsection{Stacks of Herschel/SPIRE Observations}

In order to calculate average IR luminosities and SFRs, we repeat our stacking analysis on the Herschel/SPIRE maps of the ECDFS at 250,350, and $500 \mu \mathrm{m}$. These observations are part of the Herschel Multi-tiered Extragalactic Survey (Oliver et al. 2012). The SPIRE maps have resolution elements of $17^{\prime \prime} .6,23^{\prime \prime} .9$, and $35^{\prime \prime} .1$ and reach a $1 \sigma$ depth of $1.6,1.3$, and $1.9 \mathrm{mJy}$ at 250,350 , and $500 \mu \mathrm{m}$, respectively. We use the deblended SPIRE maps described in Swinbank et al. (2014). These were obtained by constructing a catalog of IR- and radiobright galaxies based on ancillary multiwavelength data, in particular Spitzer/MIPS $24 \mu \mathrm{m}$, Very Large Array (VLA), and the catalog of precisely located sub-millimeter galaxies (SMGs) from ALESS. For each SPIRE band, a model of the image was created by assigning to each galaxy in the input catalog a SPIRE point-spread function scaled to a random flux between 0 and 1.3 times the brightest flux observed in the same region of the sky. This step was iterated until the model converged toward the observed map. For each sample of galaxies in our analysis ( $K$-selected, sBzK, ERO, and DRG), we removed all the contaminants by subtracting the best model of all the other (non-color-selected) sources in the field. These "residual maps" are used as input images for our stacking routine. The stacking strategy follows that performed for the ALESS data. Here, we focus on the $z>1$ subsamples. Our results are listed in Table 2 and shown in Figure 5.

\section{RESULTS}

\subsection{ALESS Stacks}

Figure 4 and Table 1 summarize the results of the stacking analysis applied to ALESS data. We also report the rms of the flux per pixel in the stacked maps (measured on the background at $>3^{\prime \prime}$ from the center) and the uncertainties estimated through the random stacks, as described in the previous section. These numbers allow us to gauge the robustness of a detection in our stacked images.

Clear detections are reported in most of the galaxy samples. The stacked flux of the $K_{\text {Vega }}<20$ mag sample is likely dominated by a small subsample of bright sources at $z>1$ : after removing the 10 sub-millimeter brightest sources exceeding 1.8 mJy (i.e., $10 \%$ of the sample), the stacked flux drops by a factor $\sim 3.4$. On the other hand, a positive, $5 \sigma$ signal is reported also when we adopt median stacking instead of weighted averages. The stacked flux is 1.5 times higher if we restrict our analysis only at the $z>1$ sources (we note that $\sim 73 \%$ of the $K_{\text {Vega }}<20$ mag galaxies at $z>1$ in the ALESS coverage are also classified as sBzK, EROs, or DRGs). sBzK galaxies, EROs, and DRGs all show clear detections $(>10 \sigma)$ when the whole sample is considered. As we lower the $344 \mathrm{GHz}$ flux cutoff to $1.2 \mathrm{mJy}$, DRGs still show significant detections $(\sim 5 \sigma)$, while detections at lower significance $(\sim 3 \sigma)$ are reported for sBzK and EROs. Median stacks performed without flux thresholds reveal clear detections in all the galaxy samples, suggesting that our results are robust against the contribution of bright outliers. The $z>1$ cutoff has a marginal (if any) effect on these samples. When comparing the various samples of galaxies in our analysis, we find that, for any flux or redshift cut, sBzK and DRGs exhibit the brightest $344 \mathrm{GHz}$ fluxes. This may be explained by the fact that the fraction of star-forming galaxies, as identified by the sBzK selection, is typically higher among DRGs $(\sim 35 \%)$ than among EROs ( $\sim 20 \%$, Lane et al. 2007) or $K$-selected galaxies $(\sim 9 \%$; G10).

\subsection{Herschel/SPIRE Stacks}

Figure 5 and Table 2 summarize the results of the stacking analysis applied to SPIRE data. Clear detections are reported in all the bands and for all the galaxy samples. We use these measurements to constrain the SED of dust emission in 


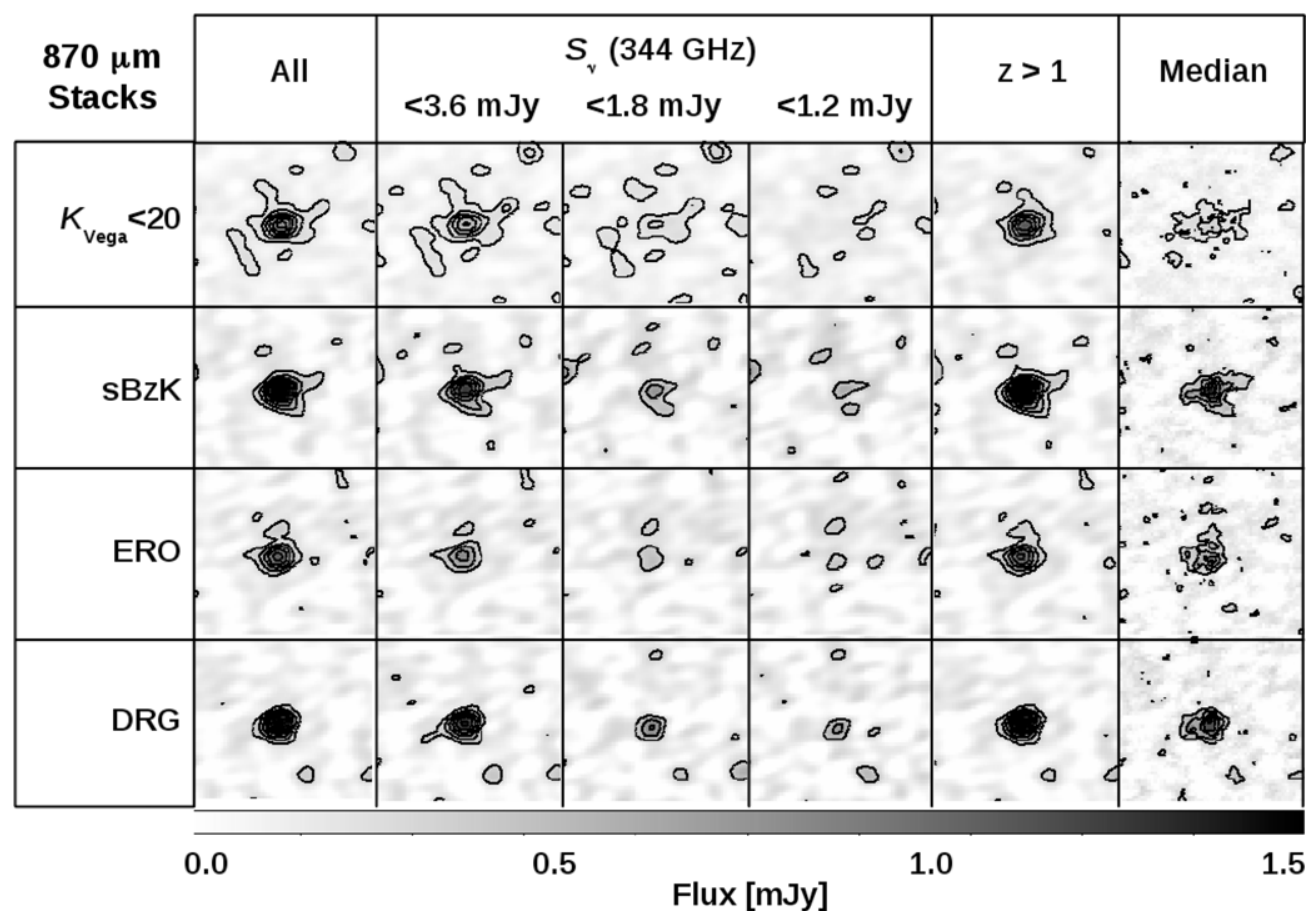

Figure 4. Postage stamps of the stacks for all the galaxy samples considered in this study. The first column refers to the weighted-average stacks of the whole samples, while the second, third, and fourth columns show the stacked results of the samples after removing sources with $S_{v}(344 \mathrm{GHz})>3.6 \mathrm{mJy}, 1.8 \mathrm{mJy}$, and $1.2 \mathrm{mJy}$, respectively. The last two columns show the stacked results for all the galaxies at $z>1$ and for the whole samples again, when median stacking is adopted instead of weighted averages. The grayscale is fixed in all the panels, while contours are $2 \sigma$ spaced and mark the significance of the detections. Each postage stamp covers an area of $10^{\prime \prime} \times 10^{\prime \prime}$. Clear detections are reported in most of the cases.

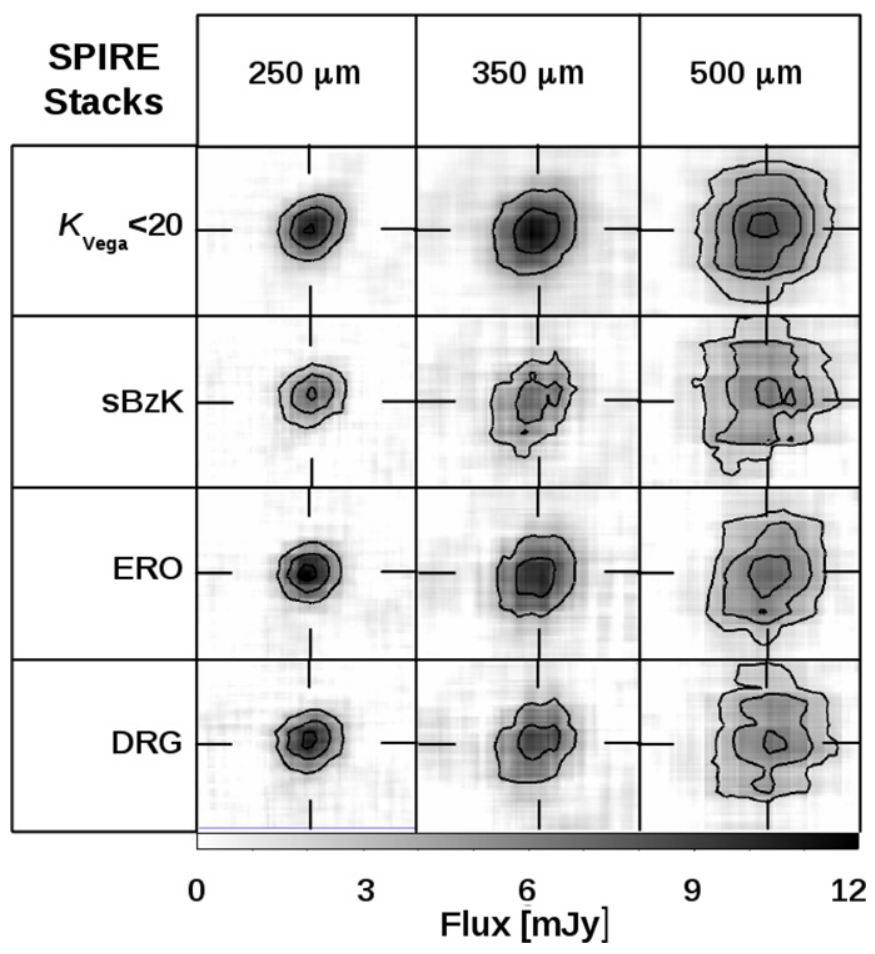

Figure 5. Postage stamps of the stacks of Herschel/SPIRE observations of the $z>1$ samples of color-selected galaxies in our analysis. Each panel is $40^{\prime \prime} \times 35^{\prime \prime}$ wide. The grayscale is fixed in all the panels, while contours are $2 \sigma$ spaced and mark the significance of the detections. All the samples show a detection in the SPIRE bands.

color-selected galaxies at $z>1$. We fit the observed SEDs as modified blackbodies $S_{v} \propto v^{\beta} B_{v}\left(T_{\text {dust }}\right)$, where $B_{v}(T)$ is the Planck function, $T_{\text {dust }}=12-60 \mathrm{~K}$ is the dust temperature, and

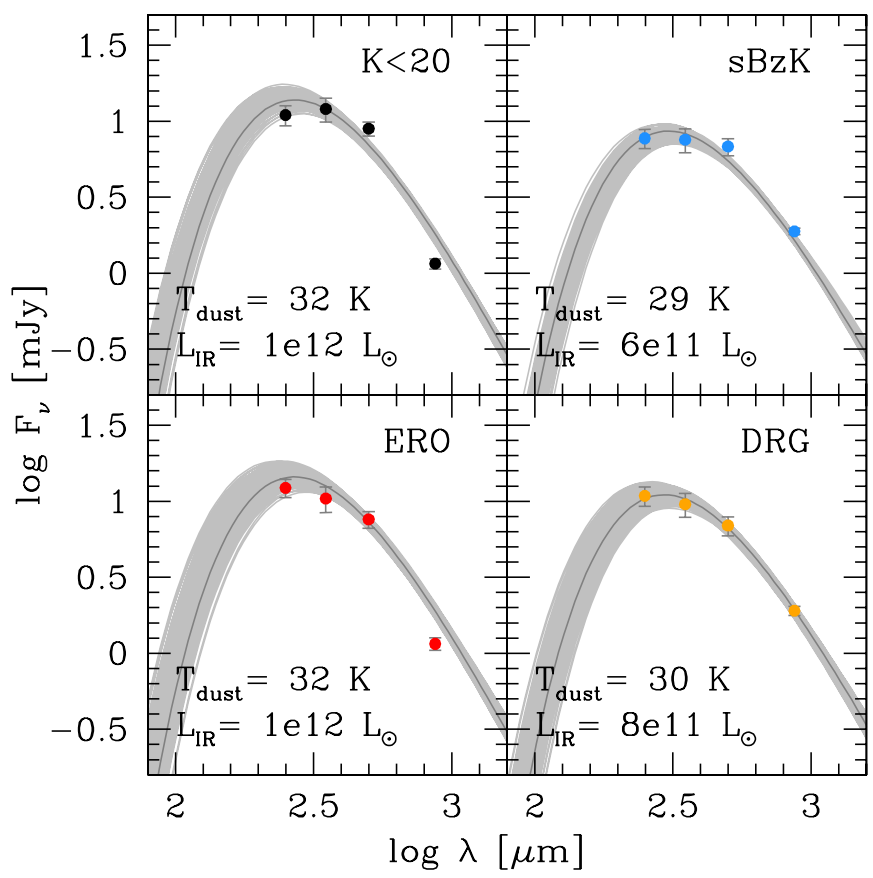

Figure 6. SEDs of $z>1$ color-selected galaxies in our analysis. Points (with error bars) show the Herschel/SPIRE and the ALESS fluxes obtained in our stacking analysis. The gray curves show the modified blackbody templates that best fit the data and the range of models that are in good agreement (within $1 \sigma$ ) with the observed constraints. The relevant best-fit parameters are also reported. (A color version of this figure is available in the online journal.)

$\beta=1.4-2.0$ sets the frequency dependence of the dust opacity (e.g., Beelen et al. 2006; Kelly et al. 2012). We find $\beta=1.6$ and $T_{\text {dust }} \approx 30 \mathrm{~K}$ in all the cases (see Figure 6). These values are in agreement with the findings of similar studies: Swinbank et al. 
Table 1

Summary of the Stacking Results

\begin{tabular}{|c|c|c|c|c|}
\hline Sample & $\begin{array}{c}\text { N. gal } \\
\text { (2) }\end{array}$ & $\begin{array}{c}\begin{array}{c}\text { Flux }^{\mathrm{a}} \\
(\mathrm{mJy})\end{array} \\
(3)\end{array}$ & $\begin{array}{c}\mathrm{rms}^{\mathrm{b}} \\
(\mathrm{mJy}) \\
(4)\end{array}$ & $\begin{array}{c}\text { Error }^{\mathrm{c}} \\
(\mathrm{mJy}) \\
(5)\end{array}$ \\
\hline \multicolumn{5}{|c|}{ All } \\
\hline$K_{\text {Vega }}<20$ & 100 & 0.78 & 0.06 & 0.06 \\
\hline sBzK & 22 & 1.88 & 0.15 & 0.11 \\
\hline ERO & 26 & 1.11 & 0.09 & 0.10 \\
\hline DRG & 20 & 1.77 & 0.13 & 0.12 \\
\hline \multicolumn{5}{|c|}{$S_{v}(344 \mathrm{GHz})<3.6 \mathrm{mJy}$} \\
\hline$K_{\text {Vega }}<20$ & 97 & 0.53 & 0.06 & 0.05 \\
\hline sBzK & 20 & 1.31 & 0.14 & 0.11 \\
\hline ERO & 25 & 0.82 & 0.09 & 0.12 \\
\hline DRG & 19 & 1.41 & 0.12 & 0.11 \\
\hline \multicolumn{5}{|c|}{$S_{v}(344 \mathrm{GHz})<1.8 \mathrm{mJy}$} \\
\hline$K_{\text {Vega }}<20$ & 90 & 0.23 & 0.05 & 0.05 \\
\hline sBzK & 16 & 0.77 & 0.14 & 0.14 \\
\hline ERO & 22 & 0.45 & 0.09 & 0.13 \\
\hline DRG & 15 & 0.89 & 0.13 & 0.14 \\
\hline \multicolumn{5}{|c|}{$S_{v}(344 \mathrm{GHz})<1.2 \mathrm{mJy}$} \\
\hline$K_{\text {Vega }}<20$ & 85 & 0.20 & 0.06 & 0.08 \\
\hline sBzK & 14 & 0.60 & 0.15 & 0.17 \\
\hline ERO & 20 & 0.39 & 0.09 & 0.13 \\
\hline$\underline{\mathrm{DRG}}$ & 13 & 0.68 & 0.11 & 0.15 \\
\hline \multicolumn{5}{|c|}{$z>1$} \\
\hline$K_{\text {Vega }}<20$ & 52 & 1.16 & 0.09 & 0.09 \\
\hline sBzK & 22 & 1.89 & 0.15 & 0.10 \\
\hline ERO & 25 & 1.15 & 0.09 & 0.11 \\
\hline$\underline{\mathrm{DRG}}$ & 19 & 1.90 & 0.13 & 0.13 \\
\hline \multicolumn{5}{|c|}{ All, median stack } \\
\hline$K_{\text {Vega }}<20$ & 100 & 0.34 & 0.07 & 0.06 \\
\hline sBzK & 22 & 1.48 & 0.15 & 0.13 \\
\hline ERO & 26 & 0.89 & 0.11 & 0.10 \\
\hline$\underline{\mathrm{DRG}}$ & 20 & 1.47 & 0.14 & 0.13 \\
\hline \multicolumn{5}{|c|}{ All, stack at uniform spatial resolution } \\
\hline$K_{\text {Vega }}<20$ & 100 & 0.79 & 0.06 & 0.06 \\
\hline $\mathrm{sBzK}$ & 22 & 1.76 & 0.15 & 0.11 \\
\hline ERO & 26 & 1.02 & 0.06 & 0.10 \\
\hline DRG & 20 & 1.55 & 0.11 & 0.12 \\
\hline \multicolumn{5}{|c|}{ Greve et al. (2010), sub-millimeter faint only } \\
\hline$K_{\text {Vega }}<20$ & 8209 & 0.18 & & 0.01 \\
\hline $\mathrm{sBzK}$ & 725 & 0.37 & & 0.04 \\
\hline ERO & 1228 & 0.29 & & 0.03 \\
\hline DRG & 720 & 0.32 & & 0.04 \\
\hline \multicolumn{5}{|c|}{ Greve et al. (2010), all } \\
\hline$K_{\text {Vega }}<20$ & 8266 & 0.22 & & 0.01 \\
\hline $\mathrm{sBzK}$ & 744 & 0.50 & & 0.04 \\
\hline ERO & 1253 & 0.39 & & 0.03 \\
\hline DRG & 737 & 0.43 & & 0.04 \\
\hline
\end{tabular}

Notes.

a Stacked $344 \mathrm{GHz}$ flux, measured as the maximum flux in a pixel within $3^{\prime \prime}$ from the central pixel.

b Pixel rms of the stacked image.

c Stack uncertainties as estimated by stacking random coordinates in each map (see Section 4.2 for details).

(2014) use combined MIPS $24 \mu \mathrm{m}$, Herschel, ALESS, and VLA data in order to constrain the dust SED in ALESS SMGs and find typical dust temperatures of $T_{\text {dust }}=20-40 \mathrm{~K}$. Bourne et al. (2012) use Herschel Astrophysical Terahertz Large Area Survey (Eales et al. 2010) data at 250,350, and $500 \mu \mathrm{m}$ to constrain the dust SED of optically selected star-forming galaxies at high $z$. They find that blue and red galaxies are well described by a modified blackbody with $\beta \approx 2$ and $T_{\text {dust }}=10-30 \mathrm{~K}$ (red galaxies having on average lower $T_{\text {dust }}$ and $L_{\text {dust }}$ ). Elbaz et al. (2011) use Herschel observations to infer typical SEDs of socalled main sequence and starbursting galaxies. They find an effective dust peak temperatures $T_{\mathrm{eff}}^{\text {peak }}$ of $31 \mathrm{~K}$ for main sequence galaxies and $40 \mathrm{~K}$ for starbursts.

IR luminosities are computed as the integral of the galaxy rest-frame SED between 8 and $1000 \mu \mathrm{m}$ (e.g., Sanders et al. 2003). These luminosities are then converted into SFRs using $\log \mathrm{SFR} /\left(M_{\odot} \mathrm{yr}^{-1}\right)=\log (1.3)-10+\log \left(L_{\mathrm{IR}} / L_{\odot}\right)($ e.g., Genzel et al. 2010). This conversion implicitly assumes a Chabrier (2003) initial mass function. We find that SFRs in our samples range between 75 and $140 M_{\odot} \mathrm{yr}^{-1}$ (see Table 2).

\subsection{Comparison with Previous Results at $870 \mu \mathrm{m}$}

Table 1 (bottom) reports the stacked fluxes obtained in G10 for their sub-millimeter faint sample (roughly comparable with our $S_{v}(344 \mathrm{GHz})<3.6 \mathrm{mJy}$ in terms of depth) and for their whole sample. We find a factor $\sim 3$ brighter fluxes than G10 for all galaxy classes, if no $S_{v}(344 \mathrm{GHz})$ cutoff is considered in our analysis. Webb et al. (2004) study the sub-millimeter properties of EROs using SCUBA. They find average $850 \mu \mathrm{m}$ fluxes of $0.40 \pm 0.07 \mathrm{mJy}$ for galaxies selected by requiring $(I-K)_{\text {Vega }}>4$ and $0.53 \pm 0.09 \mathrm{mJy}$ for galaxies with $(R-K)_{\text {Vega }}>5.3$ (i.e., EROs in our classification). Knudsen et al. (2005) obtain a $850 \mu \mathrm{m}$ average flux of $1.1 \pm 0.3 \mathrm{mJy}$ for DRGs, after stacking SCUBA observations of a cluster field (not corrected for lensing magnification). Similarly, by stacking SCUBA observations of $24 \mu \mathrm{m}$-detected BzK galaxies, Daddi et al. (2005) find an $850 \mu \mathrm{m}$ average flux of $1.0 \pm 0.1 \mathrm{mJy}$. Based on an independent stacking analysis of SCUBA observations of the Lockman Hole and the Subaru/XMM-Newton Deep Field, Takagi et al. (2007) report $850 \mu \mathrm{m}$ fluxes of $0.52 \pm 0.19 \mathrm{mJy}$, $0.52 \pm 0.16 \mathrm{mJy}$, and $0.3 \pm 0.3 \mathrm{mJy}$ for sBzK, EROs, and DRGs.

Overall, we conclude that we find brighter fluxes than most of the stacking studies performed so far at these wavelengths using single-dish data over larger fields. Possible explanations for this discrepancy are: (1) the discrepancy is an artifact, resulting from, e.g., the deblending procedures adopted in single-dish studies and (2) the discrepancy is real, i.e., the galaxies covered by ALESS pointings (defined to include the sub-mm brightest sources in the field) are intrinsically different from the general field covered in single-dish observations. The first scenario seems unlikely, given that various groups have run different analyses based on independent approaches and routines and find (to first order) consistent results. In particular, Daddi et al. (2005) and Takagi et al. (2007) do not apply any deblending.

In order to address the second scenario, we compare various properties of the galaxy samples considered in our analysis with the general field catalog and the LESS results from G10.

\subsubsection{Redshift and NIR Luminosity Distributions}

Figure 2 compares the redshift and NIR luminosity distributions of the galaxies in our sample with those of the general photometric catalog, scaled in order to have the same total number of sources. Color-selected galaxies observed in ALESS tend to have slightly higher redshifts and brighter NIR luminosities. We use a Kolmogorov-Smirnov (KS) test to assess the significance of this discrepancy. The discrepancy is marginal for the redshift distributions of $\mathrm{sBzK}$ and $\mathrm{pBzK}$ galaxies and is robust $(>3 \sigma)$ for all the other distributions. In particular, the discrepancy in the redshift distributions is dominated by a small excess 
Table 2

Stacked SPIRE Fluxes and Estimates of Dust Temperature, IR Luminosity, and SFR

\begin{tabular}{|c|c|c|c|c|c|c|c|}
\hline $\begin{array}{l}\text { Sample } \\
z>1 \\
\text { (1) }\end{array}$ & $\begin{array}{l}\langle z\rangle \\
(2)\end{array}$ & $\begin{array}{c}S_{v}(250 \mathrm{GHz}) \\
(\mathrm{mJy}) \\
(3)\end{array}$ & $\begin{array}{c}S_{v}(350 \mathrm{GHz}) \\
(\mathrm{mJy}) \\
(4)\end{array}$ & $\begin{array}{c}S_{v}(500 \mathrm{GHz}) \\
(\mathrm{mJy}) \\
(5)\end{array}$ & $\begin{array}{c}T_{\text {dust }} \\
(\mathrm{K}) \\
(6)\end{array}$ & $\begin{array}{c}\log L_{\mathrm{IR}} \\
\left(L_{\odot}\right) \\
(7)\end{array}$ & $\begin{array}{c}\mathrm{SFR} \\
\left(M_{\odot} \mathrm{yr}^{-1}\right) \\
(8)\end{array}$ \\
\hline$K_{\text {Vega }}<20$ & 1.562 & $11.0 \pm 1.7$ & $12.0 \pm 2.1$ & $8.9 \pm 1.0$ & $32_{-2}^{+7}$ & $12.01_{-0.11}^{+0.15}$ & $130_{-30}^{+50}$ \\
\hline sBzK & 1.896 & $7.7 \pm 1.1$ & $7.6 \pm 1.3$ & $6.8 \pm 0.9$ & $29_{-4}^{+4}$ & $11.75_{-0.12}^{+0.09}$ & $74_{-19}^{+17}$ \\
\hline ERO & 1.502 & $12.2 \pm 1.7$ & $10.4 \pm 2.0$ & $7.6 \pm 1.0$ & $32_{-3}^{+8}$ & $12.03_{-0.14}^{+0.19}$ & $140_{-40}^{+70}$ \\
\hline DRG & 1.792 & $10.8 \pm 1.6$ & $9.6 \pm 1.7$ & $6.9 \pm 1.0$ & $30_{-4}^{+6}$ & $11.88_{-0.12}^{+0.15}$ & $100_{-20}^{+40}$ \\
\hline
\end{tabular}

of ALESS-covered galaxies at $z \approx 2$. Almost all the galaxies classified as sBzK, EROs, and DRGs in our analysis show $M_{\mathrm{H}}<-23 \mathrm{mag}$, i.e., they are on average a factor $\sim 2.5$ times brighter than their analogs in the general field. If $H$-band and $344 \mathrm{GHz}$ luminosities are correlated in these sources (similarly to what is observed at radio wavelengths; see, e.g., Dunne et al. 2009), then the difference observed in the $H$-band luminosity distributions would explain the discrepancy between our results and those of single-dish studies.

\subsubsection{Clustering}

The ALESS pointings may be biased toward regions with most prominent overdensities of sub-millimeter bright galaxies. This is supported by various lines of evidence: (1) The brightest LESS sources appear split in multiple detections once observed at $\sim 1^{\prime \prime} 6 \times 1$.".15 resolution (Karim et al. 2013; Hodge et al. 2013), (2) SMGs tend to lie in the progenitors of moderateto-high-mass groups of galaxies $\left(M_{\text {halo }} \sim 4 \times 10^{12} M_{\odot}\right.$; see Hickox et al. 2012), and (3) pairs or multiplets of EROs are often associated with sub-mm bright regions (Ivison et al. 2002; Webb et al. 2004; Chapman et al. 2009; Wardlow et al. 2011). Aravena et al. (2010) found significant overdensities of actively starforming galaxies around three MAMBO-detected galaxies in the COSMOS field, although they do not find similar overdensities around other MAMBO sources in the same survey, suggesting that the occurrence of such structures around SMGs is about $\sim 30 \%$.

We test this scenario by comparing the clustering properties of galaxies in each class inside the ALESS coverage with those in the general photometric catalog. Figure 7 shows the number of ALESS pointings encompassing $0,1,2, \ldots$ color-selected galaxies. For each galaxy class, we extract random, equally sized sets of galaxies from the general field catalog and we compute the number of galaxies of the same class that would be covered in ALESS-sized pointings centered on such a random sample. This process is repeated 50 times, allowing us to empirically evaluate the sample variance. Results of this Monte Carlo test are shown in Figure 7 as shaded areas. In all the galaxy classes, we find consistency between the distributions of sources per pointing observed in the ALESS data and the ones of the simulations, suggesting that the sources covered in ALESS data show similar clustering properties compared with those of the general field.

\subsubsection{Radio Fluxes}

If we evaluate the contribution of the sources in our analysis to the extragalactic background light (EBL) at $344 \mathrm{GHz}$, we obtain significantly higher surface brightnesses than expected. Our analysis covered $74 \times \pi(1.2 \times 17.3 / 2)^{2} \operatorname{arcsec}^{2} \approx$ $0.00193 \mathrm{deg}^{2}$. If we divide the total flux from $K_{\text {Vega }}<20 \mathrm{mag}$ galaxies, sBzK, EROs, and DRGs by this area, we obtain 40.4 $\mathrm{Jy} \mathrm{deg}^{-2}, 21.4 \mathrm{Jy} \mathrm{deg}^{-2}, 15.0 \mathrm{Jy} \mathrm{deg}^{-2}$, and $18.3 \mathrm{Jy} \mathrm{deg}^{-2}$,

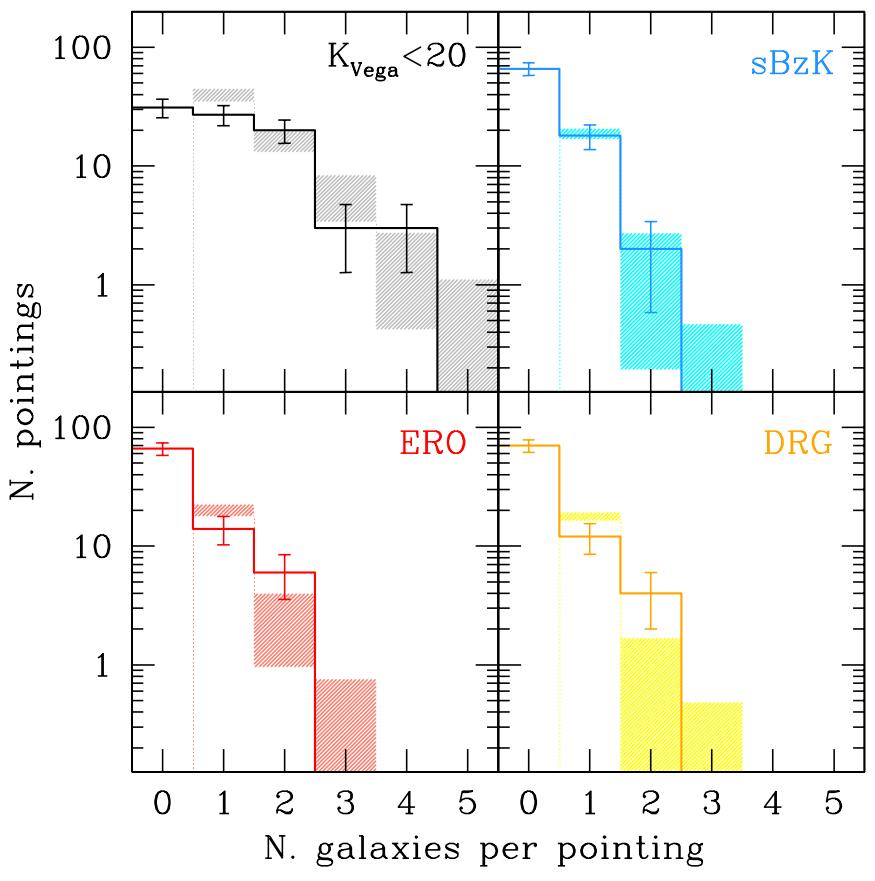

Figure 7. Comparison between the observed number of galaxies per ALESS pointing (histograms) and the expectations from random sets of galaxies of the same class derived from the general field (shaded areas). Error bars show the Poissonian uncertainties. The observed distributions are in agreement with those of the general sample, suggesting that ALESS sources have similar clustering properties as those in the whole MUSYC catalog.

(A color version of this figure is available in the online journal.)

respectively, i.e., 5-10 times higher than what was reported by G10 for the whole LESS field and close to the total EBL light (44 $\pm 15 \mathrm{Jy} \mathrm{deg}^{-2}$; see Section 5.4 in G10; note, however, that there is substantial overlap between our galaxy samples as defined by the adopted color cuts). Such a discrepancy suggests that, by survey design, ALESS pointings encompass regions of the sky that are intrinsically brighter at sub-millimeter wavelengths than the general field.

In order to test this scenario, we compare the radio $(1.4 \mathrm{GHz})$ fluxes of the sources covered by ALESS pointings with the sources in the general field. Our test relies on the observed 1.4 GHz-IR luminosity relation (Condon et al. 1982; Helou et al. 1985; Yun et al. 2001; Garrett 2002; Ivison et al. 2010; Sargent et al. 2010). Given the small differences in the redshift distributions of the photometric sample within and outside the ALESS coverage (as discussed in Section 5.3.1), if galaxies covered in ALESS pointings are intrinsically brighter at $344 \mathrm{GHz}$ than the galaxies in the general MUSYC field, we expect a similar difference to be observed also at $1.4 \mathrm{GHz}$. We base our comparison on the $1.4 \mathrm{GHz}$ map of the ECDFS obtained by Miller et al. (2008; see also Miller et al. 2013). The 


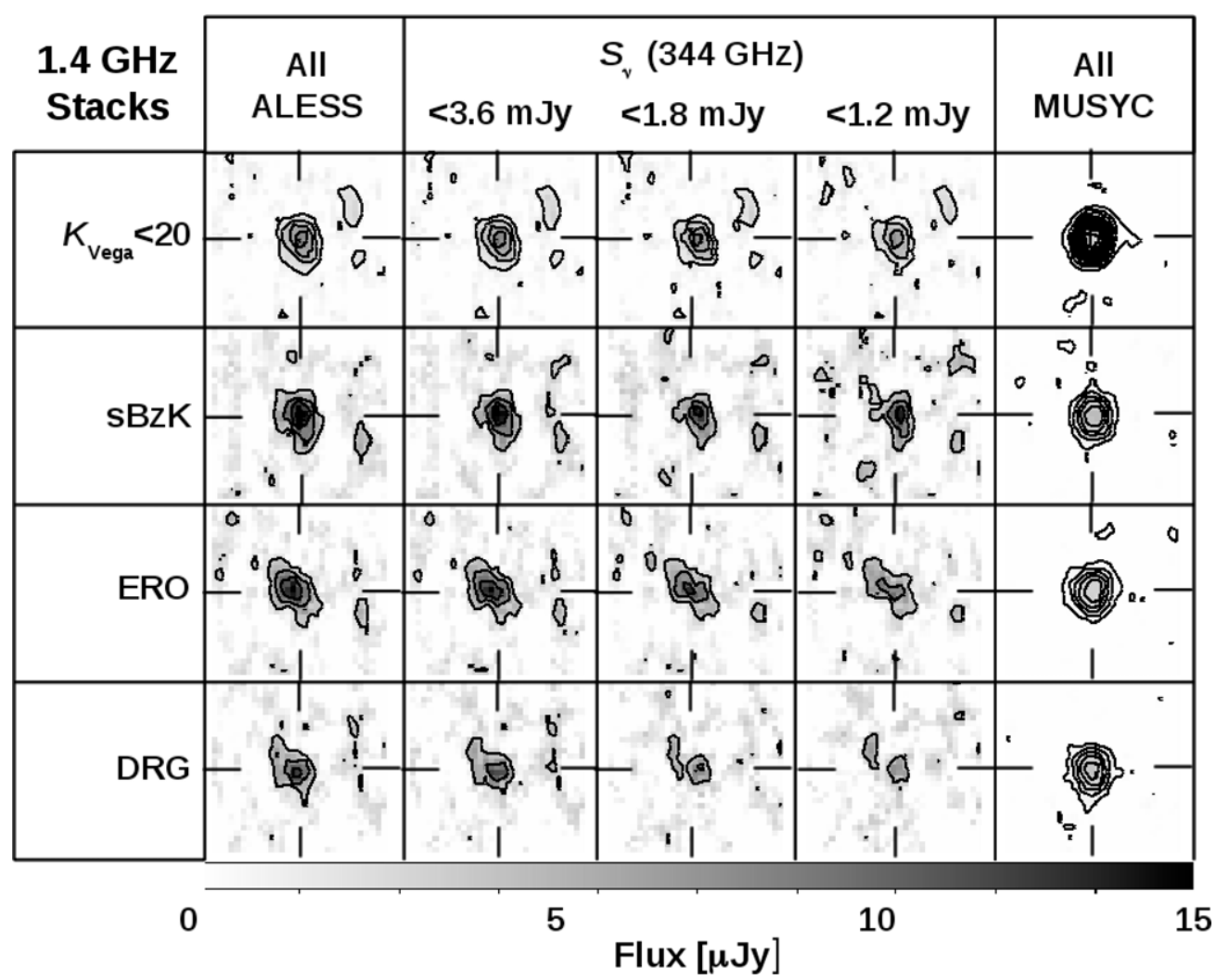

Figure 8. Postage stamps of the $1.4 \mathrm{GHz}$ median stacks of galaxies in ALESS, compared with the general MUSYC field. The first column refers to the stacks of the whole ALESS samples, while the second, third, and fourth columns show the stacked results of the samples after removing sources with $S_{v}(344 \mathrm{GHz})>3.6 \mathrm{mJy}, 1.8$ $\mathrm{mJy}$, and $1.2 \mathrm{mJy}$, respectively. The last column shows the stacked results for the all the galaxies in the MUSYC sample. The grayscale is the same in all the panels, while contours are $2 \sigma$ levels highlighting the significance of the stacked detections. Each postage stamp covers an area of $10^{\prime \prime} \times 10^{\prime \prime}$. The radio flux decreases as we lower the cutoff in $344 \mathrm{GHz}$ flux, as a result of the radio/IR correlation. The stacks from the general field show higher significance (thanks to the much larger sample sizes) but significantly lower fluxes than the sources encompassed by ALESS (see Table 3 ).

Table 3

Summary of the $1.4 \mathrm{GHz}$ Median Stacking Results

\begin{tabular}{|c|c|c|c|c|c|c|}
\hline Sample & All ALESS & & $S_{v}(344 \mathrm{GHz})$ & & All Field & ALESS Excess \\
\hline (1) & $\begin{array}{c}(\mu \mathrm{Jy}) \\
(2)\end{array}$ & $\begin{array}{c}<3.6 \mathrm{mJy} \\
(\mu \mathrm{Jy}) \\
(3)\end{array}$ & $\begin{array}{c}<1.8 \mathrm{mJy} \\
(\mu \mathrm{Jy}) \\
(4)\end{array}$ & $\begin{array}{c}<1.2 \mathrm{mJy} \\
(\mu \mathrm{Jy}) \\
(5)\end{array}$ & $\begin{array}{c}(\mu \mathrm{Jy}) \\
(6)\end{array}$ & (7) \\
\hline$K_{\text {Vega }}<20$ & $10.0 \pm 1.0$ & $9.8 \pm 1.0$ & $9.1 \pm 1.0$ & $8.2 \pm 1.0$ & $3.94 \pm 0.16$ & $2.5 \pm 0.3$ \\
\hline sBzK & $17.9 \pm 1.9$ & $16.1 \pm 2.0$ & $14.5 \pm 2.2$ & $13.8 \pm 2.2$ & $5.3 \pm 0.4$ & $3.4 \pm 0.4$ \\
\hline ERO & $15.0 \pm 1.8$ & $14.8 \pm 1.8$ & $13.2 \pm 1.8$ & $10.0 \pm 1.9$ & $3.8 \pm 0.3$ & $3.9 \pm 0.6$ \\
\hline DRG & $13.0 \pm 1.9$ & $12.4 \pm 2.1$ & $9.1 \pm 2.1$ & $8.0 \pm 2.3$ & $4.8 \pm 0.4$ & $2.7 \pm 0.5$ \\
\hline
\end{tabular}

map was obtained with the VLA in extended (A) configuration, yielding a spatial resolution of $2^{\prime \prime} .8 \times 11^{\prime \prime} .6$ over a $32^{\prime} \times 32^{\prime}$ wide region. The typical $\mathrm{rms}$ of the mosaic is $\sim 7 \mu \mathrm{Jy}_{\text {beam }}{ }^{-1}$.

In Figure 8, we show the stacks of the $1.4 \mathrm{GHz}$ images of the sources in our sample. We perform radio stacks also for the subsamples obtained with various sub-mm flux thresholds. The results are compared with the median stacks obtained for the general population of color-selected galaxies in the field. We perform both median and average stacks. The latter, however, are dominated by the contribution of a few, very bright outliers (most likely, radio-loud active galactic nuclei). Because of this, we focus only on median stacks. All the galaxy samples ( $K_{\text {Vega }}<20$ mag, sBzK, EROs, and DRGs) show clear $1.4 \mathrm{GHz}$ detections in the stacks. The significance of such detections drops as we remove sub-mm bright sources, as a result of the IRradio luminosity relation. The detection is only marginal $(3.5 \sigma)$ for DRGs as we apply the most aggressive sub-millimeter flux cut $\left(S_{v}(344 \mathrm{GHz})<1.2 \mathrm{mJy}\right)$. Remarkably, the stacks over the entire sample show considerably fainter $1.4 \mathrm{GHz}$ fluxes, by a factor 3 compared with the uncut ALESS sample (see Table 3). The latter stacked fluxes are slightly lower but still in agreement with the results of similar stacking studies at $1.4 \mathrm{GHz}$ by Dunne et al. (2009), which are clearly inconsistent with the values obtained for the ALESS-covered samples. The discrepancy in the stacked fluxes within and outside ALESS pointings is still significant even if we consider only the sources that are not individually detected in the ALESS observations. This suggests that the difference in sub-mm and radio fluxes between ALESScovered galaxies and the general sample extends to a number of galaxies in the close neighborhood of sub-millimeter galaxies.

In order to further assess the robustness of this result, in Figure 9 we show the distribution of the $1.4 \mathrm{GHz}$ fluxes of field sources within and outside the ALESS pointings. Sources in the ALESS coverage are systematically brighter. We compute 

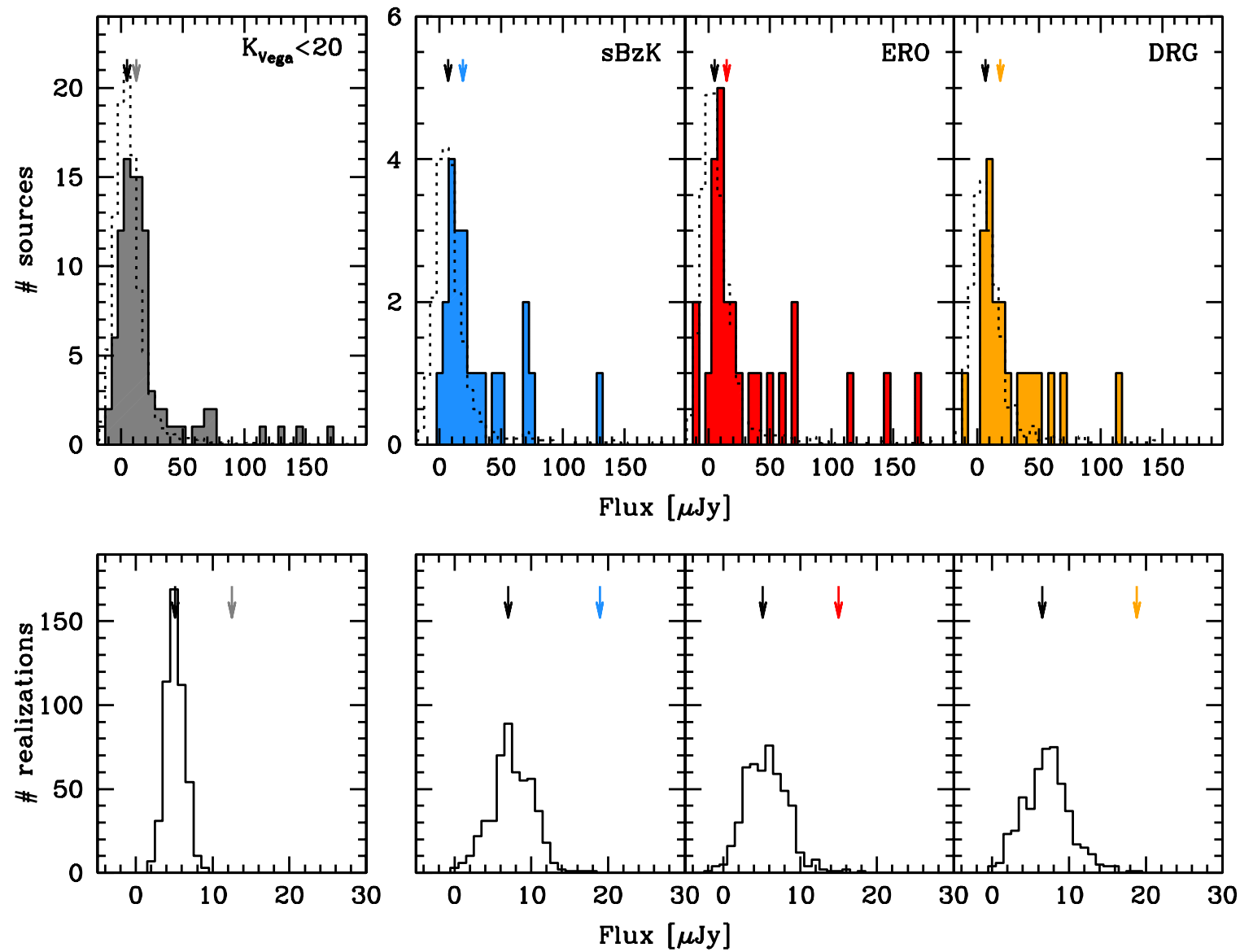

\section{$\downarrow \quad$ MUSYC}

\section{$\downarrow \vee \downarrow \vee$ ALESS}

Figure 9. Top panels: comparison of the $1.4 \mathrm{GHz}$ fluxes of the sources in our analysis (shaded histograms) with respect to the general population in the MUSYC catalog (dotted histograms). Median fluxes are labeled with downward arrows. Galaxies covered in ALESS pointings are systematically brighter at $1.4 \mathrm{GHz}$ than the general population in the MUSYC field. Bottom panels: distributions of the median $1.4 \mathrm{GHz}$ fluxes obtained by bootstrapping the MUSYC sample of galaxies, compared with the median values obtained for the ALESS galaxies (marked as arrows). The discrepancy is highly significant in all the galaxy color selections, suggesting that the discrepancy is real and does not result from an accidentaly radio-bright, randomly selected subsample of typically fainter galaxies from the MUSYC catalog.

(A color version of this figure is available in the online journal.)

median fluxes of random subsamples of the field galaxies, requiring the same sample size as the one covered in ALESS pointings. This bootstrapping approach allows us to evaluate whether the discrepancy between the general field and ALESScovered galaxies can be explained as an accidentally bright realization drawn from a typically fainter population. This test (shown in the bottom panels of Figure 9) shows that the observed median values of the $1.4 \mathrm{GHz}$ stacks of ALESS-covered galaxies represent $>4 \sigma$ outliers compared with the distribution of median stack values derived from bootstrapping the field sample. This result is confirmed via a KS test, which rules out that the distributions of $1.4 \mathrm{GHz}$ fluxes of the ALESS-covered galaxies and the general field population are drawn from the same parent distribution at $>4 \sigma$ for all the color selections.

\subsubsection{Physical Association versus Chance Projection}

Our analysis so far has shown the following: (1) we observe a discrepancy in the stacked fluxes of galaxies within ALESS pointings and the results from similar studies based on singledish observations over larger fields, (2) the discrepancy is likely driven by different sub-millimeter properties of galaxies (e.g., galaxies around SMGs tend to be brighter at sub-millimeter wavelengths than typical field galaxies with similar NIR luminosities), and (3) this hypothesis is confirmed by a similar discrepancy observed at $1.4 \mathrm{GHz}$, even after removing galaxies individually detected at sub-millimeter wavelengths. We therefore conclude that galaxies in the vicinity of SMGs (on sky) tend to be brighter than those in the general field.

We now investigate whether this effect is due to chance alignments (i.e., ALESS pointings cover regions of the sky that show projected overdensities of sub-millimeter bright galaxies) or physical associations (i.e., the sub-millimeter bright sources belong to physical overdensities around individually detected ALESS SMGs). From each of our galaxy samples, we consider as physically associated all the galaxies (including the SMGs) with a photometric redshift (from Simpson et al. 2014) consistent with the photometric redshift of the nearby $(<200 \mathrm{kpc}$ in terms of projected distance) SMG. These galaxies are highlighted with thick lines in Figure 3. Galaxies physically associated with an SMG seem to show brighter $870 \mu \mathrm{m}$ fluxes than the general color-selected samples covered by ALESS observations, although this is only tentative due to the limited sample sizes. If confirmed, this result would suggest that the discrepancy between the sub-millimeter (and radio) fluxes of color-selected galaxies inside ALESS pointings and those in the general field is intrinsic in the properties of galaxies and not simply due to chance superposition. In this scenario, galaxies spatially close to SMGs 


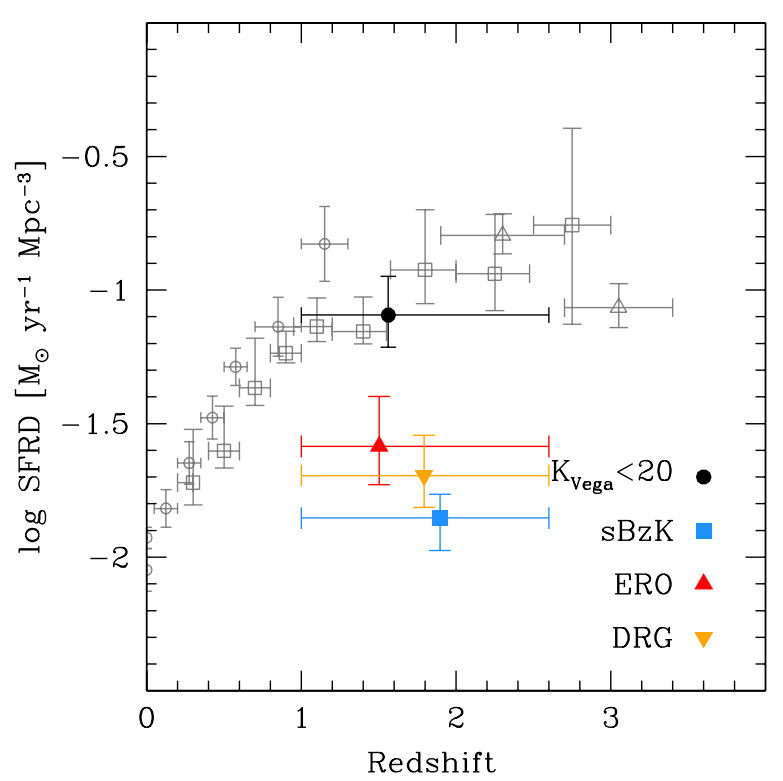

Figure 10. Cosmic SFRD as a function of redshift. Gray open points are IR/radio-based SFRD estimates from the literature: squares are from Karim et al. (2011), circles are from Rujopakarn et al. (2010), and triangles are from Reddy et al. (2008), homogenized to a Chabrier (2003) initial mass function. Filled symbols show the results from our stacking analysis based on galaxies observed in ALESS pointings. Error bars account for the formal uncertainties in the stacked fluxes, but not for the uncertainties due to model assumptions. In order to infer SFRDs, we have assumed a modified blackbody template scaled in order to match the $344 \mathrm{GHz}$ fluxes measured in our study for $z>1$ galaxies. The SFRD of $K$-selected galaxies is in agreement with previous works in the literature, although we remark that we did not correct for the excess in IR emission observed in sources within ALESS pointings. sBzK, EROs, and DRGs account for $\sim 17 \%, \sim 33 \%$, and $\sim 25 \%$ of the total SFRD from $K$-selected galaxies, respectively.

(A color version of this figure is available in the online journal.)

would show brighter sub-millimeter fluxes than similar galaxies in the field. We note, however, that the uncertainties in the photometric redshifts used in this test are large. This implies that the "physical association" flag used here may be a by-product of the similar redshift distributions of SMGs and our color-selected galaxies, rather than the result of a real physical connection.

\subsection{SFR Density}

In Figure 10, we use the estimated SFRs for the $z>1$ galaxies computed in Section 5.2 in order to put constraints on the cosmic SFRD as a function of redshift. It is claimed that SFRD smoothly increased from very high redshift until $z=1-2$, when it reached its peak $\left(\sim 0.2 M_{\odot} \mathrm{yr}^{-1} \mathrm{Mpc}^{-3}\right.$, assuming a Chabrier 2003, initial mass function). This is usually referred to as the "epoch of galaxy assembly," when roughly half of the stars in the universe were formed. At more recent cosmic times, the SFRD declined by more than an order of magnitude between $z \sim 1$ and $z=0$ (Lilly et al. 1995; Madau et al. 1996; Hopkins \& Beacom 2006; Reddy et al. 2008; Rujopakarn et al. 2010; Bouwens et al. 2010; Karim et al. 2011). We compute SFRDs associated with our sample of galaxies by multiplying the average SFRs by the number of galaxies in each sample and dividing the resulting "total" SFRs by the volume sampled in our analysis. As described in Section 3, the photometric catalog is highly complete at $K_{\text {Vega }}<20 \mathrm{mag}$. At this flux threshold, we sample galaxies up to $z=2.6$ (see Figure 2). We thus restrict our analysis to the $1<z<2.6$ range, where color selection is most effective. Our analysis covered a total of $7.0 \mathrm{arcmin}^{2}$.
The corresponding comoving volume is $34000 \mathrm{Mpc}^{3}$. Finally, we scale our SFRD estimates down in order to account for the excess in ALESS fluxes with respect to the general field (see Table 3).

We compare our results with those of other studies available in the literature, based on IR/radio SFRD measurements (Reddy et al. 2008; Rujopakarn et al. 2010; Karim et al. 2011). The SFRD derived in Rujopakarn et al. (2010) are scaled down by a factor of $1.65 \mathrm{in}$ order to account for the different initial mass function assumption.

Our estimates of the SFRD for $K$-selected galaxies are in broad agreement with previous work in the literature $\left(\approx 0.08 M_{\odot} \mathrm{yr}^{-1} \mathrm{Mpc}^{-3}\right)$. The other subsamples of galaxies (sBzK, EROs, and DRGs) account for $\sim 17 \%, \sim 33 \%$, and $\sim 25 \%$ of the total SFRD from $K$-selected galaxies, respectively (note that these samples have substantial overlap; see Section 3 ).

\section{CONCLUSIONS}

We present a stacking analysis of ALMA interferometric $344 \mathrm{GHz}$ continuum observations of high- $z$ galaxies as part of the ALESS survey. We base our stacking approach on the photometric optical/NIR catalog of field galaxies in the ECDFS. Based on their fluxes and colors, we select four classes of galaxies: $K_{\text {Vega }}<20 \mathrm{mag}$, sBzK, EROs, and DRGs. Our findings are as follows.

1. Clear $(>10 \sigma)$ detections are reported for all the galaxy classes, independently of the averaging algorithm (weighted averages versus median stacks). The detections are robust also after excluding sources bright enough to be individually detected in the original single-dish LESS observations.

2. The detection of $344 \mathrm{GHz}$ flux in $K$-selected galaxies is dominated by their high- $z$ subsample. Half of the $K_{\text {Vega }}<$ 20 mag galaxies lie at $z<1$ and show significantly fainter $344 \mathrm{GHz}$ emission than the subsample at $z>1$. In particular, a few very bright galaxies dominate the flux in the stacked maps (although a clear detection is reported also when median stacks are considered).

3. Color-selected galaxies (sBzK, EROs, and DRGs) are detected in our stacking analysis at $344 \mathrm{GHz}$, even after excluding sources that would be individually detected in the original LESS observations or in the ALESS maps.

4. We perform a similar stacking analysis on Herschel/SPIRE maps of the ECDFS and combine these findings with our ALESS results in order to constrain the shape of the dust SED in color-selected galaxies at $z>1$. We find that the IR emission of these sources is well described by a modified blackbody with $\beta=1.6$ and $T_{\text {dust }} \approx 30 \mathrm{~K}$. We infer IR luminosities of $L_{\mathrm{IR}}=(5-11) \times 10^{11} L_{\odot}$ and associated SFRs of $75-140 M_{\odot} \mathrm{yr}^{-1}$.

5. We find brighter $870 \mu \mathrm{m}$ fluxes than previously reported in similar stacking studies of this (G10) or other regions of the sky, based on single-dish observations at the same frequency. A similar discrepancy is observed if we apply our stacking approach to $1.4 \mathrm{GHz}$ observations of the ECDFS.

6. If we limit our analysis to sources with photometric redshifts consistent with a nearby SMG, we find tentative evidence that these galaxies are intrinsically brighter at $870 \mu \mathrm{m}$ than the remainder population. If confirmed, this result may be interpreted as a significant contribution of pairs of interacting galaxies to the SMG population, as has been suggested by theoretical models (e.g., Baugh 
et al. 2005). However, significantly higher precision in the redshift estimates and larger samples are mandatory in order to support this scenario.

7. When we place these SFR estimates into a cosmological context, we find that color-selected galaxies contribute to one-third to one-sixth of the cosmic SFRD at $1<z<2.6$.

Our study demonstrates the power of stacking analyses applied to interferometric data at sub-millimeter wavelengths for unveiling the properties of star-forming galaxies at high $z$. Our upcoming Cycle 1 ALMA observations of the same fields will allow us to individually detect the sources that can currently only be detected through stacking analysis and will allow us to further push down our stacking sensitivity limits.

We thank the anonymous referee for useful comments. This paper makes use of the following ALMA data: ADS/ JAO.ALMA\#2011.1.00294.S. ALMA is a partnership of ESO (representing its member states), NSF (USA), and NINS (Japan), together with NRC (Canada) and NSC and ASIAA (Taiwan), in cooperation with the Republic of Chile. The Joint ALMA Observatory is operated by ESO, AUI/NRAO, and NAOJ. This publication also makes use of data acquired with Europear Southern Observatories VLT under program ID 183.A-0666. This publication is based on data acquired with the APEX under programme IDs 078.F-9028(A), 079.F-9500(A), 080.A-3023(A), and 081.F-9500(A). APEX is a collaboration between the Max-Planck-Institut für Radioastronomie, the European Southern Observatory, and the Onsala Space Observatory. Support for R.D. was provided by the DFG priority program 1573 "The physics of the interstellar medium." I.R.S. acknowledges support from STFC (ST/IOO1573/1), a Leverhulme Fellowship, the ERC Advanced Investigator programme DUSTYGAL, and a Royal Society/Wolfson Merit Award. A.M.S. gratefully acknowledges an STFC Advanced Fellowship (ST/H005234/1). R.I. acknowledges the ERC AdG programme COSMICISM. A.K. acknowledges support from STFC as well as from the Collaborative Research Council 956 funded by the Deutsch Forschungsgemeinschaft (DFG). K.K. thanks the Swedish Research Council for support (grant 6212011-5372). The data employed in this analysis are available from the ALMA and ESO archives.

\section{REFERENCES}

Aleksić, J., Antonelli, L. A., Antoranz, P., et al. 2011, ApJ, 729, 115 Aravena, M., Bertoldi, F., Carilli, C., et al. 2010, ApJL, 708, L36 Austermann, J. E., Dunlop, J. S., Perera, T. A., et al. 2010, MNRAS, 401, 160 Barger, A. J., Cowie, L. L., \& Sanders, D. B. 1999, ApJL, 518, L5 Baugh, C. M., Lacey, C. G., Frenk, C. S., et al. 2005, MNRAS, 356, 1191 Beelen, A., Cox, P., Benford, D. J., et al. 2006, ApJ, 642, 694 Bell, E. F. 2003, ApJ, 586, 794

Blain, A. W., Smail, I., Ivison, R. J., Kneib, J.-P., \& Frayer, D. T. 2002, PhR, 369,111

Borys, C., Chapman, S., Halpern, M., \& Scott, D. 2003, MNRAS, 344, 385 Bourne, N., Maddox, S. J., Dunne, L., et al. 2012, MNRAS, 421, 3027 Bouwens, R. J., Illingworth, G. D., Oesch, P. A., et al. 2010, ApJL, 709, L133 Boyle, B. J., Cornwell, T. J., Middelberg, E., et al. 2007, MNRAS, 376, 1182 Brinchmann, J., Charlot, S., White, S. D. M., et al. 2004, MNRAS, 351, 1151 Chabrier, G. 2003, PASP, 115, 763

Chapman, S. C., Blain, A., Ibata, R., et al. 2009, ApJ, 691, 560

Chapman, S. C., Blain, A. W., Ivison, R. J., \& Smail, I. R. 2003, Natur, 422, 695

Chapman, S. C., Blain, A. W., Smail, I., \& Ivison, R. J. 2005, ApJ, 622, 772

Chaudhary, P., Brusa, M., Hasinger, G., et al. 2012, A\&A, 537, 6

Condon, J. J. 1992, ARA\&A, 30, 575

Condon, J. J., Condon, M. A., Gisler, G., \& Puschell, J. J. 1982, ApJ, 252, 102
Coppin, K., Chapin, E. L., Mortier, A. M. J., et al. 2006, MNRAS, 372, 1621 da Cunha, E., Walter, F., Decarli, R., et al. 2013, ApJ, 765, 9 Daddi, E., Cimatti, A., Renzini, A., et al. 2004, ApJ, 617, 746 Daddi, E., Dickinson, M., Chary, R., et al. 2005, ApJ, 626, 680 Daddi, E., Dickinson, M., Morrison, G., et al. 2007, ApJ, 670, 156 Damen, M., Labbé, I., van Dokkum, P. G., et al. 2011, ApJ, 727, 1 Dole, H., Lagache, G., Puget, J.-L., et al. 2006, A\&A, 451, 417 Dunne, L., Ivison, R. J., Maddox, S., et al. 2009, MNRAS, 394, 3 Eeles, S., Raymond, G., Roseboom, I. G., et al. 2010, A\&A, 518, L23 Elbaz, D., Dickinson, M., Hwang, H. S., et al. 2011, A\&A, 533, 119 Elston, R., Rieke, G. H., \& Rieke, M. J. 1988, ApJL, 331, L77 Fabello, S., Catinella, B., Giovanelli, R., et al. 2011, MNRAS, 411, 993 Franx, M., Labbé, I., Rudnick, G., et al. 2003, ApJL, 587, L79 Garrett, M. A. 2002, A\&A, 384, L19

Gawiser, E., van Dokkum, P. G., Herrera, D., et al. 2006, ApJS, 162, 1 Genzel, R., Tacconi, L. J., Gracia-Carpio, J., et al. 2010, MNRAS, 407, 2091 George, M. R., Leauthaud, A., Bundy, K., et al. 2012, ApJ, 757, 2

Giacconi, R., Rosati, P., Tozzi, P., et al. 2001, ApJ, 551, 624 González, V., Bouwens, R. J., Labbé, I., et al. 2012, ApJ, 755, 148

Greve, T. R., Weiß, A., Walter, F., et al. 2010, ApJ, 719, 483 Hatsukade, B., Iono, D., Akiyama, T., et al. 2010, ApJ, 711, 974 Helou, G., Soifer, B. T., \& Rowan-Robinson, M. 1985, ApJL, 298, L7

Hickox, R. C., Wardlow, J. L., Smail, I., et al. 2012, MNRAS, 421, 284 Hodge, J. A., Becker, R. H., White, R. L., \& de Vries, W. H. 2008, AJ, 136,1097

Hodge, J. A., Karim, A., Smail, I., et al. 2013, ApJ, 768, 91

Hodge, J. A., Zeimann, G. R., Becker, R. H., \& White, R. L. 2009, AJ, 138, 900

Hopkins, A. M., \& Beacom, J. F. 2006, ApJ, 651, 142

Hsieh, B.-C., Wang, W.-H., Hsieh, C.-C., et al. 2012, ApJS, 203, 23

Ivison, R. J., Greve, T. R., Dunlop, J. S., et al. 2007, MNRAS, 380, 199

Ivison, R. J., Greve, T. R., Smail, I., et al. 2002, MNRAS, 337, 1

Ivison, R. J., Magnelli, B., Ibar, E., et al. 2010, A\&A, 518, L31

Karim, A., Schinnerer, E., Martínez-Sansigre, A., et al. 2011, ApJ, 730, 61

Karim, A., Swinbank, A. M., Hodge, J. A., et al. 2013, MNRAS, 432, 2

Kelly, B. C., Shetty, R., Stutz, A. M., et al. 2012, ApJ, 752, 55

Kennicutt, R. C. 1998, ARA\&A, 36, 189

Kewley, L. J., Dopita, M. A., Sutherland, R. S., Heisler, C. A., \& Trevena, J. 2001, ApJ, 556, 121

Knudsen, K. K., Neri, R., Kneib, J.-P., \& van der Werf, P. P. 2009, A\&A, 496, 45

Knudsen, K. K., van der Werf, P., Franx, M., et al. 2005, ApJL, 632, L9

Kurczynski, P., \& Gawiser, E. 2010, AJ, 139, 1592

Lane, K. P., Almaini, O., Foucaud, S., et al. 2007, MNRAS, 379, L25

Leroy, A. K., Bigiel, F., de Blok, W. J. G., et al. 2012, AJ, 144, 3

Lilly, S. J., Tresse, L., Hammer, F., Crampton, D., \& Le Fèvre, O. 1995, ApJ, 455, 108

Madau, P., Ferguson, H. C., Dickinson, M. E., et al. 1996, MNRAS, 283, 1388

Matsuda, Y., Yamada, T., Hayashino, T., et al. 2012, MNRAS, 425, 878

Miller, N. A., Bonzini, M., Fomalont, E. B., et al. 2013, ApJS, 205, 13

Miller, N. A., Fomalont, E. B., Kellermann, K. I., et al. 2008, ApJS, 179, 114

Moncelsi, L., Ade, P. A. R., Chapin, E. L., et al. 2011, ApJ, 727, 83

Murphy, E. J., Bremseth, J., Mason, B. S., et al. 2012, ApJ, 761, 97

Oke, J. B. 1974, ApJS, 27, 21

Oliver, S. J., Bock, J., Altieri, B., et al. 2012, MNRAS, 424, 1614

Reddy, N. A., Steidel, C. C., Pettini, M., et al. 2008, ApJS, 175, 48

Rujopakarn, W., Eisenstein, D. J., Rieke, G. H., et al. 2010, ApJ, 718, 1171

Salim, S., Rich, R. M., Charlot, S., et al. 2007, ApJS, 173, 267

Sanders, D. B., Mazzarella, J. M., Kim, D.-C., Surace, J. A., \& Soifer, B. T. 2003, AJ, 126, 1607

Sargent, M. T., Schinnerer, E., Murphy, E., et al. 2010, ApJL, 714, L190

Simpson, J. M., Swinbank, A. M., Smail, I., et al. 2014, ApJ, submitted (arXiv:1310.6363)

Solomon, P. M., \& Vanden Bout, P. A. 2005, ARA\&A, 43, 677

Swinbank, A. M., Simpson, J. M., Smail, I., et al. 2014, MNRAS, submitted (arXiv:1310.6362)

Swinbank, A. M., Smail, I., Longmore, S., et al. 2010, Natur, 464, 733

Takagi, T., Mortier, A. M. J., Shimasaku, K., et al. 2007, MNRAS, 381, 1154

Taylor, E. N., Franx, M., van Dokkum, P. G., et al. 2009, ApJS, 183, 295

Walter, F., Decarli, R., Carilli, C., et al. 2012, Natur, 486, 233

Walter, F., Riechers, D., Cox, P., et al. 2009, Natur, 457, 699

Wardlow, J. L., Smail, I., Coppin, K. E. K., et al. 2011, MNRAS, 415, 1479

Webb, T. M. A., Brodwin, M., Eales, S., \& Lilly, S. J. 2004, ApJ, 605, 645

Weiß, A., Kovács, A., Coppin, K., et al. 2009, ApJ, 707, 1201

Yun, M. S., \& Carilli, C. L. 2002, ApJ, 568, 88

Yun, M. S., Reddy, N. A., \& Condon, J. J. 2001, ApJ, 554, 803

Zibetti, S., Ménard, B., Nestor, D. B., et al. 2007, ApJ, 658, 161 